\title{
Fibrés principaux sur les corps valués henséliens
}

\author{
Ofer Gabber, Philippe Gille et Laurent Moret-Bailly
}

\begin{abstract}
Let $K$ be the field of fractions of a henselian valuation ring $A$. Assume that the completion $\widehat{K}$ is a separable extension of $K$. Let $Y$ be a $K$-variety, let $G$ be an algebraic group over $K$, and let $f: X \rightarrow Y$ be a $G$-torsor over $Y$. We consider the induced map $X(K) \rightarrow Y(K)$, which is continuous for the topologies deduced from the valuation. If $I$ denotes the image of this map, we prove that $I$ is locally closed in $Y(K)$; moreover, the induced surjection $X(K) \rightarrow I$ is a principal bundle with group $G(K)$ (also topologized by the valuation).
\end{abstract}

\section{RÉSUMÉ}

Soit $K$ le corps des fractions d'un anneau de valuation hensélien $A$. On suppose que le complété $\widehat{K}$ est une extension séparable de $K$. Soient $Y$ une $K$-variété, $G$ un $K$-groupe algébrique et $f: X \rightarrow Y$ un $G$-torseur au-dessus de $Y$. On considère l'application induite $X(K) \rightarrow Y(K)$, continue pour les topologies déduites de la valuation. Si $I$ désigne son image, nous montrons que $I$ est localement fermée dans $Y(K)$; de plus la surjection induite $X(K) \rightarrow I$ est un fibré principal sous le groupe topologique $G(K)$.

\section{Table des matières}

1 Introduction

1.1 Notations . . . . . . . . . . . . . . . . . . . . . . . 574

1.3 Plan de la démonstration. . . . . . . . . . . . . . . . . . . . 575

1.4 Application aux espaces homogènes. . . . . . . . . . . . . . . . . 576

1.5 Application aux orbites. . . . . . . . . . . . . . . . 576

1.6 Plan de l'article. . . . . . . . . . . . . . . . . . . 576

1.7 Conventions . . . . . . . . . . . . . . . . . . . . . 577

2 Actions de groupes algébriques: rappels et compléments $\quad \mathbf{5 7 8}$

2.1 Orbites dans un espace algébrique à groupe d'opérateurs . . . . . . . . . 578

2.2 Quotient par une action libre . . . . . . . . . . . . . . . . . . 579

2.3 Produit contracté d'un torseur et d'un espace à opérateurs . . . . . . . . . 580

2.4 Le plus grand sous-groupe lisse d'un groupe algébrique . . . . . . . . . . 581

2.5 Compactifications partielles dans les schémas de Hilbert ponctuels . . . 583

Received 3 June 2014, accepted in final form 4 June 2014.

2010 Mathematics Subject Classification 20G25, 14L30, 11D88.

Keywords: local fields, valuation fields, algebraic groups, homogeneous spaces, torsors, compactifications This journal is (c) Foundation Compositio Mathematica 2014. This article is distributed with Open Access under the terms of the Creative Commons Attribution Non-Commercial License, which permits non-commercial reuse, distribution, and reproduction in any medium, provided that the original work is properly cited. For commercial re-use, please contact the Foundation Compositio Mathematica.

Le second auteur a bénéficié du soutien du projet ANR Gatho, ANR-12-BS01-0005. 


\section{Ofer Gabber, Philippe Gille et Laurent Moret-Bailly}

3 Corps topologiquement henséliens; le cas des torseurs sous un groupe lisse

3.1 Variétés sur un corps topologique; corps topologiquement henséliens . . 585

3.2 Extension aux espaces algébriques . . . . . . . . . . . . . . . 587

3.3 Corps valués henséliens: utilisation de modèles entiers . . . . . . . . . . 589

3.4 Torseurs sous un groupe lisse . . . . . . . . . . . . . . . . . . . . . 591

3.5 Corps valués henséliens: approximation faible et applications . . . . . . . 592

4 Corps valués admissibles; le cas d'un groupe $G$ tel que $G_{\text {red }}^{\circ}$ soit lisse 595

4.1 Corps valués admissibles: généralités . . . . . . . . . . . . . . . . 595

4.2 Le théorème d'approximation fort; applications . . . . . . . . . . . . . . 596

4.3 Groupes $G$ tels que $G_{\text {red }}^{\circ}$ soit lisse . . . . . . . . . . . . . . . . 598

5 Un théorème de compactification $\quad \mathbf{5 9 9}$

5.3 Démonstration du théorème 5.2 : dévissage . . . . . . . . . . . . . . . 600

5.4 Démonstration du théorème 5.2 : construction et fin . . . . . . . . . . . . 602

6 Démonstration du théorème $1.2 \quad 603$

7 Exemples et compléments $\quad \mathbf{6 0 5}$

7.1 Un exemple d'orbite topologique non fermée . . . . . . . . . . . . . 605

7.2 Contre-exemples sur un corps valué hensélien non admissible . . . . . . . 605

7.3 Espaces non localement séparés. . . . . . . . . . . . . . . . 606

7.4 Cas d'un schéma en groupes non constant . . . . . . . . . . . . . . 608

$\begin{array}{ll}\text { References } & \mathbf{6 1 0}\end{array}$

\section{Introduction}

\subsection{Notations}

Soit $K$ le corps des fractions d'un anneau de valuation $A$; on notera $v$ la valuation associée et $\Gamma$ son groupe. La donnée de $v$ détermine une structure de corps topologique séparé sur $K$; nous supposerons toujours qu'il n'est pas discret, c'est-à-dire que $\Gamma \neq 0$. On notera $\widehat{K}$ le complété de $K$.

Dans cette introduction, nous supposerons $(K, v)$ admissible, au sens suivant.

1.1.1 Définition. Avec les notations ci-dessus, on dit que $(K, v)$ (ou $A$ ) est admissible si $A$ est hensélien et si l'extension $\widehat{K} / K$ est séparable.

Pour toute $K$-variété (c'est-à-dire tout $K$-schéma de type fini) $X$, on note $X_{\text {top l'ensemble }}$ $X(K)$ muni de la topologie déduite de la topologie de $K$. Tout $K$-morphisme $f: X \rightarrow Y$ de $K$-variétés induit une application continue $f_{\text {top }}: X_{\text {top }} \rightarrow Y_{\text {top }}$.

Nous nous intéressons dans cet article au cas où un $K$-groupe algébrique $G$ agit à droite sur $X$ et où $f: X \rightarrow Y$ est un $G$-torseur pour cette action. Noter qu'alors le groupe topologique $G_{\text {top }}$ agit librement et continûment sur $X_{\text {top }}$ et que l'on a une bijection continue $\overline{f_{\text {top }}}: X_{\text {top }} / G(K) \longrightarrow$ $I:=\operatorname{Im}\left(f_{\text {top }}\right)$. L'objet de ce travail est l'étude topologique des applications $f_{\text {top }}$ et $\overline{f_{\text {top }}}$.

Il est en fait naturel (et à certains égards plus simple) de considérer la situation plus générale où $X$ et $Y$ sont des espaces algébriques de type fini (et quasi-séparés) sur $K$, que nous appellerons dorénavant « $K$-espaces ». Tout d'abord, un $G$-torseur sur une $K$-variété $Y$ - défini, comme il se doit, comme faisceau pour la topologie fidèlement plate de présentation finie (fppf) sur $Y$ n'est pas nécessairement un schéma (en-dehors du cas important où $G$ est affine), alors que c'est automatiquement un $K$-espace, selon un théorème d'Artin; à partir de là, il devient également 


\section{FibRÉS PRINCIPAUX SUR LES CORPS VALUÉS HENSÉLIENS}

judicieux d'envisager des $G$-torseurs $X \rightarrow Y$ où $X$ et $Y$ sont des $K$-espaces, afin de travailler dans une catégorie stable par les opérations usuelles, produits fibrés notamment.

D'autre part, si un $K$-groupe algébrique $G$ opère librement à droite sur une $K$-variété $X$, le faisceau fppf quotient $X / G$ est toujours un $K$-espace (voir 2.2) mais, de nouveau, n'est pas nécessairement une $K$-variété.

Ces considérations nous ont conduits à formuler systématiquement nos résultats dans le cadre des $K$-espaces; si le plan général des démonstrations n'en est pas affecté, il nous a fallu revenir sur des résultats bien connus pour les schémas mais dont l'extension aux espaces algébriques n'est pas suffisamment documentée : voir par exemple 3.2 pour la définition et les propriétés de $X_{\text {top }}$ lorsque $X$ est un $K$-espace. (En ce qui concerne $G$, rappelons qu'un espace algébrique en groupes quasi-séparé de type fini sur un corps est toujours un schéma [Art69, Lemma 4.2]).

Notre résultat principal est le suivant.

1.2 Théorème. Soient $(K, v)$ un corps valué admissible, $G$ un $K$-groupe algébrique (c'est-à-dire un $K$-schéma en groupes de type fini), $Y$ un $K$-espace, $f: X \rightarrow Y$ un $G$-torseur au-dessus de $Y$. Définissons $f_{\text {top }}: X_{\text {top }} \rightarrow Y_{\text {top }}$ et $\overline{f_{\text {top }}}: X_{\text {top }} / G(K) \longrightarrow I:=\operatorname{Im}\left(f_{\text {top }}\right)$ comme ci-dessus. Alors :

(1) en tant que sous-espace de $Y_{\text {top }}, I$ est :

(a) localement fermé (dans tous les cas);

(b) ouvert et fermé si $G$ est lisse ou si $K$ est parfait;

(c) fermé si $G$ satisfait la condition (*) (voir 2.4.3), et en particulier si $G_{\text {red }}^{\circ}$ est lisse, ou si $G^{\circ}$ est commutatif, ou si $G$ est de rang réductif nul (en d'autres termes, si $G_{\bar{K}}$ n'a pas de sous-tore non trivial).

(2) L'application $f_{\text {top }}$ est ouverte sur son image $I$; en particulier, la bijection $\overline{f_{\text {top }}}$ est un homéomorphisme.

(3) Si $Y$ est localement séparé (par exemple une variété, cf. 1.7), alors $f_{\text {top }}$ fait de $X_{\text {top }}$ un

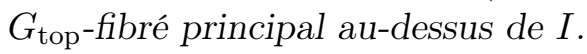

\subsection{Plan de la démonstration.}

Pour ne pas alourdir l'introduction, nous supposons ici que $Y$ est localement séparé, par exemple une $K$-variété.

Le cas, sans doute bien connu, où le groupe $G$ est lisse est traité au $\S 3$. Dans le cas général, on note $G^{\natural}$ le plus grand $K$-sous-groupe lisse de $G$ (§2.4). On décompose alors le $G$-torseur $f: X \rightarrow Y$ en

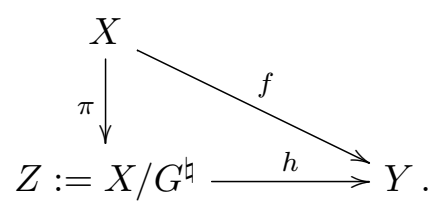

Le morphisme $\pi$ est un $G^{\natural}$-torseur et $h: Z \rightarrow Y$ est une «fibration en $G / G^{\natural}$ ». Les propriétés de $G^{\natural}$ impliquent que l'application $h_{K}: Z(K) \rightarrow Y(K)$ est injective. D'un point de vue topologique, on a donc le diagramme

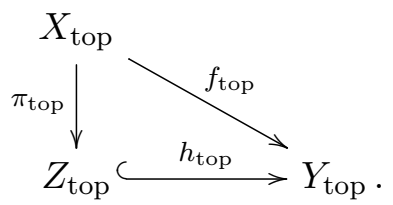




\section{Ofer Gabber, Philippe Gille et Laurent Moret-Bailly}

D'après le cas lisse $(\S 3.4), \pi_{\text {top }}$ fait de $X_{\text {top }}$ un $G_{\text {top }}^{\natural}$-fibré principal sur l'image de $\pi_{\text {top }}$, qui est ouverte et fermée dans $Z_{\text {top }}$.

L'application $h_{\text {top }}$ est nettement plus délicate à analyser. Les deux outils clés pour cette étude sont :

- le théorème d'approximation de Greenberg (généralisé aux corps valués admissibles dans $[\mathrm{MB} 12 \mathrm{~b}])$;

- la «bonne compactification » $(\S 5)$ de $G / G^{\natural}$, et la compactification relative de $h$ qui s'en déduit.

On montre ainsi que $h_{\text {top }}$ est un homéomorphisme sur son image, et que celle-ci s'écrit $F_{1} \backslash F_{2}$ pour des fermés remarquables $F_{1}, F_{2}$ de $Y_{\text {top }}$ (lemme 6.1).

\subsection{Application aux espaces homogènes.}

Un cas particulier important est celui où $X=H$ est un $K$-groupe algébrique contenant $G$ comme sous-groupe, et où $f: H \rightarrow Y:=H / G$ est le morphisme de passage au quotient. L'image $I$ est alors l'orbite sous $H(K)$ de la classe neutre $y_{0} \in Y(K)$ dans l'ensemble $Y(K)=(H / G)(K)$ : on voit donc par 1.2 qu'elle est localement fermée dans $Y_{\text {top }}$ et qu'elle s'identifie (avec sa topologie) à l'espace quotient $H_{\mathrm{top}} / G(K)$ (avec, le cas échéant, les compléments (1)(b), (1)(c) de l'énoncé) et que $H_{\text {top }}$ est même un $G_{\text {top }}$-fibré principal au-dessus de $I$.

Si de plus $G$ est distingué dans $H$, il en résulte que $I$ est toujours fermée puisque c'est un sous-groupe localement fermé du groupe topologique $Y_{\text {top }}$.

\subsection{Application aux orbites.}

Considérons un $K$-groupe algébrique $H$ opérant à gauche sur une $K$-variété $S$ (ou plus généralement sur un $K$-espace); soit $s_{0}$ un point de $S(K)$, de stabilisateur $G \subset H$. On sait alors que le morphisme d'orbite $\omega_{s_{0}}: h \mapsto h . s_{0}$ se factorise en

$$
H \stackrel{f}{\longrightarrow} H / G \stackrel{\sim}{\longrightarrow} Y \hookrightarrow S
$$

où $f$ est le morphisme canonique, où la deuxième flèche est un isomorphisme et la troisième une immersion (voir [DG70, III.3.5.2] lorsque $S$ est une variété, et 2.1 plus bas pour les $k$-espaces). D'autre part, l'image $I$ de $H(K)$ par $\omega_{s_{0}}$ est évidemment l'orbite de $s_{0}$ sous l'action de $H(K)$, dans l'espace $S(K)$, et le stabilisateur de $s_{0}$ pour cette action est $G(K)$.

Le théorème 1.2 nous dit donc que cette orbite est localement fermée dans $Y_{\text {top }}$ et donc dans $S_{\text {top }}$, et que la surjection continue canonique $H_{\text {top }} \nrightarrow I$ est une fibration principale de groupe $G_{\text {top }}$; en particulier elle induit un homéomorphisme de $H_{\text {top }} / G(K)$ sur $I$.

Lorsque $K$ est un corps local, on trouve en partie ce résultat dans l'article [BZ76]; plus précisément, les auteurs montrent que $I$ est localement fermée dans $S_{\text {top }}$ et s'identifie topologiquement à $H_{\text {top }} / G(K)$ (dans [BZ76], ces assertions sont dispersées entre 1.5, 1.6 et 6.8).

\subsection{Plan de l'article.}

Le $\S 2$ est consacré à des rappels sur les groupes algébriques : produit contracté d'un torseur et d'un schéma à opérateurs, propriétés du plus grand sous-groupe lisse d'un groupe algébrique $G$ et la technique de compactification partielle d'espaces homogènes via les schémas de Hilbert ponctuels.

$\mathrm{Au} \S 3$, après des rappels sur les variétés sur un corps topologique, on introduit la notion de corps topologiquement hensélien, caractérisée par la validité du théorème des fonctions implicites ; 


\section{FibRÉS PRINCIPAUX SUR LES CORPS VALUÉS HENSÉLIENS}

le cas des torseurs sous un groupe lisse (cf. $1.2(1)(b))$ est valable dans ce cadre, et est établi au $\S 3.4$.

C'est aussi sur un corps topologiquement hensélien que le foncteur $X \mapsto X_{\text {top }}$ (des variétés vers les espaces topologiques) s'étend aux espaces algébriques avec de bonnes propriétés.

$\mathrm{Au} \S 4$, nous donnons quelques propriétés des corps valués admissibles, notamment le théorème d'approximation fort (généralisant celui de Greenberg). Nous en déduisons un résultat topologique (théorème 4.2.3) concernant les morphismes propres, qui sera essentiel dans la preuve de 1.2. Un cas particulier de 4.2.3 est le fait (assez facile à démontrer directement) que si $K$ est admissible et si $f$ est un morphisme $f i n i$ de $K$-variétés, l'application induite $f_{\text {top }}$ est fermée (4.2.6).

Le $\S 5$, indépendant des précédents, est consacré à la démonstration d'un théorème de compactification dû à Gabber (5.2, cas particulier d'un théorème annoncé dans [Gab12]) : soient $G$ un groupe algébrique sur un corps $k$ quelconque, et $G^{\natural}$ son plus grand sous-groupe lisse. Alors le $G$-espace homogène $G / G^{\natural}$ admet une compactification équivariante dont le seul point à corps résiduel séparable sur $k$ est l'origine.

Le $\S 6$ contient la démonstration, esquissée plus haut, des parties restantes (1)(a) et (2) du théorème 1.2. Enfin le $\S 7$ contient divers (contre-)exemples et compléments.

1.6.1 Remarque. Des questions voisines (dans le cas d'une valuation complète de rang 1) sont abordées dans [BaT13]; il est à noter cependant que l'assertion (1) (a) du théorème 1 de cet article est contredite par l'exemple donné au $§ 7.1$ du présent travail.

\subsection{Conventions}

Si $k$ est un corps, une $k$-variété est un $k$-schéma de type fini; un $k$-groupe algébrique est un $k$-schéma en groupes de type fini ; un $k$-espace est un $k$-espace algébrique de type fini et quasiséparé. On renvoie à [Knu71] pour les propriétés des espaces algébriques. Rappelons simplement ici qu'un $k$-espace $X$ est localement séparé si le monomorphisme diagonal $X \rightarrow X \times_{k} X$ est une immersion; cette condition est toujours vérifiée si $X$ est une variété [EGAI, (5.1.2)].

Si $k^{\prime}$ est une extension finie de $k$, et $V$ un $k^{\prime}$-espace, nous noterons $\prod_{k^{\prime} / k}(V)$ sa restriction de Weil à $k$. On rappelle qu'en tant que foncteur sur la catégorie des $k$-espaces $T$, elle est définie par

$$
\left(\prod_{k^{\prime} / k}(V)\right)(T):=V\left(T \times_{k} k^{\prime}\right)
$$

et qu'elle est représentable par un $k$-espace ([Con12, Lemma 5.10] ou [Ols06, Theorem 1.5]), et par une $k$-variété lorsque $V$ est une $k$-variété quasi-projective ([BLR90, Theorem 7.6/4 et Proposition 7.6/5] ou [CGP10, A.5.8]).

Sauf mention contraire, la cohomologie des faisceaux utilisée est la cohomologie fppf. En particulier, si $G$ est un $k$-groupe algébrique et $Y$ un $k$-espace, $\mathrm{H}^{1}(Y, G)$ désigne l'ensemble des classes d'isomorphie de $G_{Y}$-torseurs pour la topologie fppf.

Une application continue $f: X \rightarrow Y$ entre espaces topologiques est dite stricte si la topologie induite sur $\operatorname{Im}(f) \subset Y$ coïncide avec la topologie quotient déduite de la surjection canonique $X \rightarrow$ $\operatorname{Im}(f)$ (en d'autres termes, si la bijection canonique $\operatorname{Coim}(f) \rightarrow \operatorname{Im}(f)$ est un homéomorphisme).

Une application continue $f$ entre espaces topologiques est dite propre si elle est universellement fermée (conformément à [Bou71, I, §10, $\mathrm{n}^{\circ} 1$, définition 1], nous ne faisons pas d'hypothèse de séparation). Il revient au même de dire que $f$ est fermée à fibres quasi-compactes [Bou71, I, §10, $\mathrm{n}^{\circ} 2$, théorème 1$]$. 


\section{Ofer Gabber, Philippe Gille et Laurent Moret-Bailly}

\section{Actions de groupes algébriques : rappels et compléments}

Dans cette section, $k$ désigne un corps; on fixe une clôture algébrique $\bar{k}$ de $k$, et l'on note $k_{s} \subset \bar{k}$ la clôture séparable correspondante.

\subsection{Orbites dans un espace algébrique à groupe d'opérateurs}

On fixe un $k$-groupe algébrique $G$. Soit $Y$ un $k$-faisceau fppf muni d'une action à gauche de $G$. Nous dirons que l'action est transitive (ou que $Y$ est transitif sous $G$ ) si $Y \rightarrow \operatorname{Spec}(k)$ est un épimorphisme (de façon équivalente, il existe une extension finie $k^{\prime}$ de $k$ telle que $\left.Y\left(k^{\prime}\right) \neq \emptyset\right)$ et si le morphisme

$$
\begin{aligned}
G \times Y & \longrightarrow Y \times Y \\
(g, y) & \longmapsto(g . y, y)
\end{aligned}
$$

est un épimorphisme.

2.1.1 ThÉORÈme. Soit $Y$ un $k$-faisceau fppf muni d'une action transitive de $G$. On suppose qu'il existe une extension finie $k^{\prime}$ de $k$ et un élément y de $Y\left(k^{\prime}\right)$ tel que le sous-faisceau de $G_{k^{\prime}}$ stabilisateur de $y$ soit un sous-schéma en groupes fermé de $G_{k^{\prime}}$. Alors :

(1) $Y$ est un $k$-schéma quasi-projectif.

(2) Soit $X$ un $k$-espace muni d'une action à gauche de $G$, et soit $f: Y \rightarrow X$ un morphisme $G$-équivariant. Alors $f$ se factorise de manière essentiellement unique en $Y \stackrel{\pi}{\rightarrow} Z \stackrel{j}{\rightarrow} X$, où :

(i) $Z$ est un $k$-schéma quasi-projectif;

(ii) $\pi$ est fidèlement plat;

(iii) $j$ est une immersion.

Démonstration. (1) Le $k^{\prime}$-faisceau $Y \times_{k} k^{\prime}$ obtenu par changement de base est clairement isomorphe à $G / H$, où $H$ est le stabilisateur de $y^{\prime}$. C'est donc un $k^{\prime}$-schéma quasi-projectif : si $G$ est lisse et connexe ce résultat est dû à Chow [Cho57], et le cas général s'en déduit, cf. [Ray70, VI.2.6]. L'assertion (1) en résulte par [SGA1, VIII, 7.6] (descente de schéma quasi-projectifs par un morphisme fini localement libre).

Montrons l'assertion (2). L'unicité est claire, puisque $Z$ est nécessairement le sous-faisceau de $X$ image de $f$. Montrons d'abord que $Z$, ainsi défini, est un $k$-schéma quasi-projectif : choisissons une extension finie $k^{\prime}$ de $k$ et un point $y \in Y\left(k^{\prime}\right)$. Alors le stabilisateur de $z:=\pi(y)$ est aussi celui de $j(z)$ ( $j$ est un monomorphisme) et est donc un sous-schéma en groupes fermé de $G_{k^{\prime}}$ : on conclut par l'assertion (1).

Pour voir que $j$ est une immersion et que $\pi$ est fidèlement plat, on peut donc, par descente fidèlement plate, supposer $k$ algébriquement clos, ce que nous ferons désormais. Fixons encore un point $y \in Y(k)$, de stabilisateur $S \subset G$. Alors $Y$ s'identifie à $G / S$ et $Z$ à $G / H$ où $H$ est le stabilisateur de $z=f(y) ; \pi$ s'identifie à la projection $G / S \rightarrow G / H$, qui est fidèlement plate. Il reste à voir que $j: Z \hookrightarrow X$ est une immersion, $Z$ étant l'orbite de $z$ dans $X$.

Si $X$ est une variété, cela résulte de [DG70, III.3.5.2]; nous allons nous ramener à ce cas.

Supposons d'abord $G$ réduit, de sorte que $G / H$ l'est également. On sait [Knu71, II, Proposition 6.7] que $X$ admet un plus grand sous-espace ouvert $U$ qui est un schéma, et que $U$ est dense dans $X$. Il est clair que $U$ est stable par l'action du groupe $G(k)$, et donc par l'action de $G$ puisque $k$ est algébriquement clos. Donc, si $z \in U(k), j$ se factorise par $U$ et l'assertion résulte du cas des variétés. Sinon, comme $G$ est réduit, $j$ se factorise par le sous-espace réduit $X^{\prime}$ complémentaire 


\section{FibRÉS PRINCIPAUX SUR LES CORPS VALUÉS HENSÉLIENS}

de $U$, qui est également stable par $G$. La densité implique que $\operatorname{dim}\left(X^{\prime}\right)<\operatorname{dim}(X)$ et l'on conclut par récurrence sur la dimension.

Dans le cas général, posons $Z=G / H$. Comme $Z_{\text {red }}=G_{\text {red }} /\left(G_{\text {red }} \cap H\right)$, le cas précédent montre que la restriction de $j$ à $Z_{\text {red }}$ est une immersion. On conclut donc par le lemme 2.1.1.1 qui suit.

2.1.1.1 Lemme. Soit $j: Z \rightarrow X$ un monomorphisme de type fini d'espaces algébriques. On suppose que la restriction $j_{0}$ de $j$ à $Z_{\text {red }}$ est une immersion (resp. une immersion fermée). Alors $j$ est une immersion (resp. une immersion fermée).

Démonstration. La restriction $j_{0}$ se décompose en une immersion fermée $Z_{\text {red }} \rightarrow U$ suivie d'une immersion ouverte $U \rightarrow X$; comme $j$ se factorise automatiquement par $U$, on peut remplacer $X$ par $U$, et il suffit de traiter le cas d'une immersion fermée. Alors $j$ est propre puisque $j_{0}$ l'est; d'autre part $j$ est séparé et quasi-fini donc est quasi-affine d'après [LMB00, A.2] (ou [Knu71, II, 6.15] lorsque $j$ est de présentation finie). Un morphisme propre et quasi-affine est fini, et un monomorphisme fini est une immersion fermée.

2.1.1.2 Remarque. Comme nous l'a signalé un rapporteur, on peut aussi utiliser le critère valuatif [Moc99, I, Corollary 2.13, p. 102] pour démontrer 2.1.1.1.

2.1.2 Corollaire. Soit $X$ un k-espace muni d'une action à gauche de $G$, et soit $Y$ un sousfaisceau de $X$, stable par $G$ et transitif. Alors $Y$ est un sous-espace localement fermé de $X$, et un $k$-schéma quasi-projectif.

Démonstration. Il existe une extension finie $k^{\prime}$ de $k$ et un point $y \in Y\left(k^{\prime}\right)$. Le stabilisateur de $y$ est le même dans $Y$ et dans $X$; c'est donc un sous-schéma en groupes fermé de $G$, et la conclusion résulte facilement de 2.1.1.

2.1.3 Définition. Soit $X$ un $k$-espace muni d'une action à gauche de $G$. Une $k$-orbite de $X$ sous $G$ est un sous-espace de $X$, stable par $G$ et transitif.

2.1.4 Remarques. Soit $X$ un $k$-espace muni d'une action à gauche de $G$.

(i) (orbite d'un $k$-point) Fixons un point $x \in X(k)$. Le morphisme $i: \operatorname{Spec}(k) \rightarrow X$ correspondant est alors une immersion fermée (vérification laissée au lecteur) de sorte que le stabilisateur $G_{x}$ est un $k$-sous-groupe fermé de $G$. On considère le morphisme d'orbite $f_{x}: G \rightarrow X, g \mapsto g . x$. Alors le $k$-schéma quotient $G / G_{x}$ représente le faisceau fppf image $T_{x}$ de $f_{x}$. Le théorème 2.1.1 montre que $T_{x} \rightarrow X$ est une immersion, ainsi $T_{x}$ est une $k$-orbite de $X$ sous $G$.

(ii) Si $X$ est une $k$-variété, la notion de $k$-orbite sous $G$ coïncide avec celle de [BLR90, $§ 10.2$, définition 10.4] : ceci résulte du corollaire 2.1.2.

\subsection{Quotient par une action libre}

Soit $G$ un $k$-groupe algébrique opérant à droite sur un $k$-espace $X$. On note $\alpha: X \times_{k} G \rightarrow X$ l'action en question. On suppose que l'action $\alpha$ est libre, c'est-à-dire que le morphisme

$$
\begin{aligned}
\rho=\left(\mathrm{pr}_{1}, \alpha\right): X \times_{k} G & \longrightarrow X \times_{k} X \\
(x, g) & \longmapsto(x, x . g)
\end{aligned}
$$

est un monomorphisme.

ThÉorème. Sous les hypothèses ci-dessus, le faisceau quotient $X / G$ est un $k$-espace, et la projection naturelle $\pi: X \rightarrow X / G$ fait de $X$ un $G$-torseur au-dessus de $X / G$. 
De plus, $X / G$ est localement séparé (resp. séparé) si et seulement si $\rho: X \times_{k} G \rightarrow X \times_{k} X$ est une immersion (resp. une immersion fermée).

Démonstration. Par définition, $X / G$ est le quotient (au sens des faisceaux fppf) de $X$ par la relation d'équivalence $R \subset X \times_{k} X$ image de $\rho$. Comme $\rho$ est un monomorphisme, $R$ s'identifie via $\rho$ à $X \times{ }_{k} G$ et l'on voit donc que $R$ est une relation d'équivalence plate. Le fait que $X / G$ soit un $k$-espace résulte donc de [Art74, Corollary 6.3]. D'autre part nos hypothèses impliquent que les diagrammes
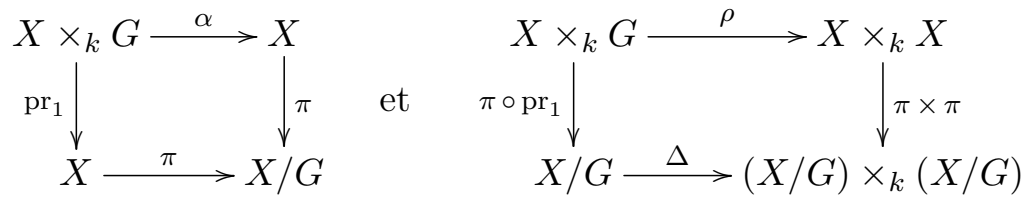

sont cartésiens, $\Delta$ désignant le morphisme diagonal. On en déduit, grâce au premier diagramme, que $X$ est un $G$-torseur sur $X / G$; le second donne les assertions de séparation (remarquer que les flèches verticales sont fidèlement plates).

\subsection{Produit contracté d'un torseur et d'un espace à opérateurs}

2.3.1 Produits contractés. Soit $G$ un faisceau en groupes sur $\operatorname{Spec}(k)$, opérant à droite sur un $k$-faisceau $X$ et à gauche sur un $k$-faisceau $U$. Rappelons que le produit contracté $X \wedge{ }_{\wedge}^{G} U$ est le faisceau quotient de $X \times_{k} U$ par l'action à droite de $G$ donnée par $((x, u), g) \mapsto\left(x g, g^{-1} u\right)$.

Nous supposerons dans la suite que $G$ est un $k$-groupe algébrique, que $X$ et $U$ sont des $k$ espaces, et de plus que $G$ opère librement sur $X$, de sorte que $X$ est un $G$-torseur au-dessus du $k$-espace $Y:=X / G(2.2)$. Dans ce cas, $G$ opère aussi librement sur $X \times_{k} U$ via l'action ci-dessus, de sorte que $X \stackrel{G}{\wedge} U$ est un $k$-espace. En outre, on a un morphisme canonique $X \stackrel{G}{\wedge} U \rightarrow Y$, localement isomorphe (pour la topologie fppf) à la première projection $Y \times U \rightarrow Y$; cette construction commute à tout changement de $k$-espace de base $Y^{\prime} \rightarrow Y$.

2.3.2 Torsion. Notons $G^{\prime}$ le $Y$-faisceau $\underline{\mathrm{Aut}}_{G}(X / Y)$ des automorphismes du $G_{Y}$-torseur $X$ : c'est une forme tordue de $G_{Y}$, qui opère naturellement à gauche sur $X$ par $Y$-morphismes, et aussi sur $X \stackrel{G}{\wedge} Z$ pour tout $Y$-faisceau $Z$ muni d'une action à gauche de $G_{Y}$. On obtient de cette façon un foncteur ( «torsion par $X »$ ) de la catégorie des $Y$-faisceaux avec action à gauche de $G_{Y}$ vers celle des $Y$-faisceaux avec action à gauche de $G^{\prime}$. Ce foncteur est une équivalence de catégories [Gir71, III, remarque 1.6.7].

Lorsque $Y=\operatorname{Spec}(k)$, il s'ensuit formellement, en particulier, que l'on a une bijection naturelle entre les $k$-orbites de $U$ sous $G$ et les $k$-orbites de $X \stackrel{G}{\wedge} U$ sous $G^{\prime}$.

2.3.3 Faisceaux inversibles. Si $L$ est un fibré en droites $G$-linéarisé sur $U$, alors $M:=X \wedge{ }_{\wedge}^{G} L$ est de façon naturelle un fibré en droites $G^{\prime}$-linéarisé sur $X{ }^{G} U$. Supposons en outre que $Y$ et $U$ soient des variétés, et que $L$ soit ample sur $U$; alors $M$ est ample sur $X{ }^{G} U$ relativement à $Y$, et en particulier $X \wedge{ }_{\wedge}^{G} U$ est un $k$-schéma quasi-projectif sur $Y$ (et même projectif si $U$ est projectif sur $k$ ) ; voir [BLR90, §10.2, Lemma 6]. 


\section{FIBRÉS PRINCIPAUX SUR LES CORPS VALUÉS HENSÉLIENS}

\subsection{Le plus grand sous-groupe lisse d'un groupe algébrique}

Rappelons [CGP10, Lemma C.4.1] qu'une $k$-variété $X$ admet un sous-schéma fermé canonique, que nous noterons $X^{\natural}$, caractérisé comme le plus petit des sous-schémas fermés $X^{\prime}$ tels que $X^{\prime}(L)=X(L)$ pour toute extension séparable $L$ de $k$; on peut construire $X^{\natural}$ comme l'adhérence schématique de l'ensemble des points de $X$ à corps résiduel séparable (resp. fini séparable) sur $k$. Ce sous-schéma est fonctoriel en $X$ (pour les morphismes de $k$-variétés), et sa formation commute aux extensions séparables des scalaires et aux produits de $k$-variétés; en particulier, si $X$ est un $k$-groupe algébrique, alors $X^{\natural}$ est un sous-groupe lisse de $X$ (et donc son plus grand sous-groupe lisse); on prendra garde qu'il n'est pas nécessairement distingué. Ceci étant, si on considère un produit semi-direct $N \rtimes G$ de $k$-groupes algébriques, l'isomorphisme $N^{\natural} \times_{k} G^{\natural} \stackrel{\sim}{\longrightarrow}(N \times G)^{\natural}$ de $k$-schémas indique que l'action de $G^{\natural}$ sur $N$ stabilise $N^{\natural}$ et que l'on a un isomorphisme $N^{\natural} \rtimes_{k} G^{\natural} \stackrel{\sim}{\longrightarrow}\left(N \rtimes_{k} G\right)^{\natural}$.

2.4.1 Proposition. Soient $G$ un k-groupe algébrique et $T$ un $G$-torseur à droite sur Spec $(k)$. Notons $\varepsilon \in\left(G / G^{\natural}\right)(k)$ la classe neutre, $Q$ le quotient $T / G^{\natural}$ et $L$ une extension séparable de $k$. Alors :

(1) on a $\left(G / G^{\natural}\right)(L)=\left(G / G^{\natural}\right)(k)=\{\varepsilon\}$.

(2) On a les équivalences :

$$
Q\left(k_{s}\right) \neq \emptyset \Longleftrightarrow T\left(k_{s}\right) \neq \emptyset \Longleftrightarrow \operatorname{Card}\left(Q\left(k_{s}\right)\right)=1 \Longleftrightarrow \operatorname{Card}(Q(k))=1 .
$$

Démonstration. (1) Soit $x \in\left(G / G^{\natural}\right)(L)$ : comme le morphisme canonique $\pi: G \rightarrow G / G^{\natural}$ est lisse et surjectif, la fibre $G_{x}$ de $\pi$ en $x$ admet des points à corps résiduel séparable sur $L$, et donc sur $k$; ces points appartiennent donc à $G_{L}^{\natural}$, donc $x=\varepsilon$.

(2) Supposons $Q\left(k_{s}\right)$ non vide; considérant le morphisme lisse surjectif $T \rightarrow Q$, on voit comme en (1) que $T\left(k_{s}\right) \neq \emptyset$. Ensuite, si $T\left(k_{s}\right) \neq \emptyset$, alors $T_{k_{s}} \cong G_{k_{s}}$ et donc $Q_{k_{s}} \cong\left(G / G^{\natural}\right)_{k_{s}}$, d'où Card $\left(Q\left(k_{s}\right)\right)=1$ d'après l'assertion $(1)$.

Si $Q\left(k_{s}\right)$ est réduit à un point, alors ce point est $k$-rationnel par descente, donc Card $(Q(k))=1$. Enfin, l'implication Card $(Q(k))=1 \Rightarrow Q\left(k_{s}\right) \neq \emptyset$ est triviale.

2.4.2 Corollaire. Soient $G$ un $k$-groupe algébrique, $Y$ une $k$-variété et $f: X \rightarrow Y$ un $G$-torseur sur $Y$. Posons $Z=X / G^{\natural}$ et soit $h: Z \rightarrow Y$ le morphisme canonique. Alors, pour toute extension séparable $k^{\prime}$ de $k$, l'application $Z\left(k^{\prime}\right) \rightarrow Y\left(k^{\prime}\right)$ induite par $f$ est injective.

Démonstration. Cela résulte de 2.4.1(2) appliqué aux fibres de $h$.

La condition suivante intervient dans les compactifications de groupes [Gab12] :

2.4.3 DÉfinition. On dit qu'un $k$-groupe algébrique $G$ satisfait la condition $(*)$ si tous les $\bar{k}$-tores de $G_{\bar{k}}$ sont des $\bar{k}$-tores de $\left(G^{\natural}\right)_{\bar{k}}$.

Cette propriété est «insensible » aux extensions séparables de $k$; plus précisément :

2.4.4 Lemme. Soit $G$ un k-groupe algébrique, et soit $L$ une extension de $k$. Si $G$ vérifie $(*)$, le $L$-groupe $G_{L}$ vérifie $(*)$. La réciproque est vraie si l'extension $L / k$ est séparable.

Démonstration. On sait que $\left(G^{\natural}\right)_{L} \subset\left(G_{L}\right)^{\natural}$, avec égalité lorsque $L / k$ est séparable. Les assertions résultent donc du lemme 2.4.4.1 qui suit, appliqué avec $H=G^{\natural}$. 


\section{Ofer Gabber, Philippe Gille et Laurent Moret-Bailly}

2.4.4.1 Lemme. Pour un k-groupe algébrique $G$ et un sous-groupe lisse $H$ de $G$, désignons par $*(k, H, G)$ la propriété «tout sous- $\bar{k}$-tore de $G_{\bar{k}}$ est contenu dans $H_{\bar{k}}$ ».

Alors, si $L$ est une extension de $k$, les propriétés $*(k, H, G)$ et $*\left(L, H_{L}, G_{L}\right)$ sont équivalentes.

Démonstration. Il est trivial que $*\left(L, H_{L}, G_{L}\right)$ entraîne $*(k, H, G)$.

Pour la réciproque on peut supposer $k$ algébriquement clos. Supposons que $*\left(L, H_{L}, G_{L}\right)$ ne soit pas vérifiée : soient donc $\bar{L}$ une clôture algébrique de $L$ et $T$ un sous-tore de $G_{\bar{L}}$ non contenu dans $H_{\bar{L}}$. Alors il existe un $k$-schéma $Y$ affine, intègre et de type fini, dont le corps des fonctions est une sous-extension (de type fini) de $\bar{L}$, et un sous- $Y$-tore $\mathscr{T}$ de $G_{Y}$ qui n'est pas contenu dans $H_{Y}$. Comme $k$ est algébriquement clos, il existe un point $y \in Y(k)$ tel que la fibre $\mathscr{T}_{y}$ de $\mathscr{T}$ en $y$ (vue comme sous-tore de $G$ ) ne soit pas contenue dans $H$. Ainsi, $*(k, H, G)$ n'est pas satisfaite.

2.4.5 Lemme. Soit $G$ un k-groupe algébrique.

(1) Si $G$ est lisse, unipotent ou commutatif, il vérifie $(*)$.

(2) Soit $G^{\prime}$ un $k$-sous-groupe de $G$ contenant la composante neutre $G^{\text {Ło }}$ de $G^{\natural}$. Si $G$ vérifie (*), alors $G^{\prime}$ vérifie $(*)$.

(3) Soit $G^{\prime}$ un $k$-sous-groupe de $G$ tel que $G / G^{\prime}$ soit fini. Pour que $G$ vérifie (*), il faut et il suffit que $G^{\prime}$ vérifie $(*)$.

(4) On suppose que $G$ est un $k$-sous-groupe distingué d'un $k$-groupe lisse $H$. Alors $G$ vérifie (*).

Démonstration. Pour l'assertion (1), le cas lisse et le cas unipotent sont évidents; pour le cas commutatif, remarquer que l'unique tore maximal de $G_{\bar{k}}$ est défini sur $k$ [CGP10, C.4.4] et donc contenu dans $G^{\natural}$. L'assertion (2) est immédiate, ainsi que l'assertion (3) car $G_{\bar{k}}$ et $G_{\bar{k}}^{\prime}$ ont les mêmes sous-tores.

Montrons l'assertion (4). Le fait que $G$ soit distingué dans $H$, et $H$ lisse, entraîne que $G^{\natural}$ est un sous-groupe distingué de $H$ : en effet $H\left(k_{s}\right)$ normalise $G_{k_{s}}^{\natural}$ et est dense dans $H$ puisque $H$ est lisse, donc le normalisateur de $G^{\natural}$ dans $H$ est égal à $H$.

Sans perte de généralité, nous pouvons supposer $k$ séparablement clos. En particulier le groupe lisse $H$ admet un $k$-tore maximal $E_{0}$ et $T_{0}=\left(E_{0} \cap G\right)_{\text {red }}^{0}$ est un $k$-tore maximal de $G$ et a fortiori de $G^{\natural}$. Soit $T$ un tore maximal de $G_{\bar{k}}$. Alors $T=\left(E \cap G_{\bar{k}}\right)_{\text {red }}^{0}$ pour un tore maximal $E$ de $H_{\bar{k}}$. On sait qu'il existe $h \in H(\bar{k})$ satisfaisant $E=h E_{0, \bar{k}} h^{-1}$ [CGP10, C.4.5(1)]. Ainsi $T=h T_{0, \bar{k}} h^{-1} \subset$ $h\left(G^{\natural}\right)_{\bar{k}} h^{-1}=\left(G^{\natural}\right)_{\bar{k}}$.

2.4.6 Remarque. Il existe des $k$-groupes résolubles de dimension 1 qui ne satisfont pas la condition $(*)$, voir $\S 7.1$.

Dans ce travail, la condition $(*)$ interviendra via le lemme suivant, variante du lemme de Rosenlicht.

2.4.7 Lemme. Soit $\bar{k}$ une clôture algébrique de $k$. Soit $\Gamma$ un $k$-groupe algébrique affine opérant sur une $k$-variété quasi-affine $Z$. Soit $s \in Z(k)$, et soit $S \subset \Gamma$ le stabilisateur de $s$. On suppose que tous les $\bar{k}$-tores de $\Gamma$ sont contenus dans $S$. Alors l'orbite $\Gamma$.s de $s$ est fermée dans $Z$.

Démonstration. On peut supposer $k$ algébriquement clos et $\Gamma$ lisse (remplacer $\Gamma$ par $\Gamma_{\text {red }}$ ne change pas l'orbite ensemblistement). Alors $\Gamma . s$ est réunion finie de transformés de $\Gamma^{\circ} . s$ par des éléments de $\Gamma(k)$ : il suffit donc de voir que l'orbite $\Gamma^{\circ} . s$ sous $\Gamma^{\circ}$ est fermée. On supposera donc aussi $\Gamma$ connexe. Remplaçant $Z$ par l'adhérence de $\Gamma . s$, on supposera $\Gamma$.s dense dans $Z$. 


\section{FibRÉS PRINCIPAUX SUR LES CORPS VALUÉS HENSÉLIENS}

Dans ces conditions, le sous-groupe $\Gamma_{t}$ de $\Gamma$ engendré par ses sous-tores (qui est distingué) opère trivialement sur $Z$, de sorte que l'action se factorise par $U:=\Gamma / \Gamma_{t}$, groupe affine lisse connexe qui n'admet pas de sous-tore non trivial [CGP10, A.2.8] et est donc unipotent. Vu l'hypothèse quasi-affine, le lemme de Rosenlicht [SGA3, XVII.5.7.3] montre alors que les orbites de $U$ dans $Z$ sont fermées.

\subsection{Compactifications partielles dans les schémas de Hilbert ponctuels}

2.5.1 Notations. On fixe une $k$-algèbre finie $k^{\prime}$, de dimension $d \geqslant 1$, et un $k^{\prime}$-espace algébrique $Q^{\prime}$, séparé et de type fini. On note $\sigma: Q^{\prime} \rightarrow \operatorname{Spec}\left(k^{\prime}\right)$ le morphisme structural et :

- $V$ le $k$-espace induit $Q^{\prime} \rightarrow \operatorname{Spec}\left(k^{\prime}\right) \rightarrow \operatorname{Spec}(k)$ (de sorte que $\sigma$ peut être vu comme un morphisme de $k$-espaces $\left.V \stackrel{\sigma}{\rightarrow} \operatorname{Spec}\left(k^{\prime}\right)\right)$;

- $W=\prod_{k^{\prime} / k} Q^{\prime}$ le foncteur de restriction de Weil de $Q^{\prime}$ relativement à $k^{\prime} / k$.

Pour tout $k$-schéma $S$, on a donc

$$
W(S)=Q^{\prime}\left(k^{\prime} \times_{k} S\right) .
$$

On considère d'autre part le foncteur de Hilbert $V^{[d]}=\mathrm{Hilb}_{V / k}^{d}$ des sous- $k$-espaces (fermés) finis de longueur $d$ de $V$. Explicitement, pour tout $k$-schéma $S$, on a

$$
\begin{aligned}
& V^{[d]}(S)=\left\{\text { sous-espaces } Z \subset V \times_{k} S,\right. \\
& \text { finis localement libres de rang } d \text { sur } S\} \text {. }
\end{aligned}
$$

Un tel $Z$ est automatiquement un schéma (affine sur $S$ ); on lui associe le $S$-morphisme composé

$$
\varphi_{Z}: Z \hookrightarrow V \times_{k} S \stackrel{\sigma \times_{k} \operatorname{Id}_{S}}{\longrightarrow} k^{\prime} \times_{k} S .
$$

Noter que $\varphi_{Z}$ est un morphisme de $S$-schémas finis localement libres de même rang $d$.

2.5.1.1 Remarque. Nous considérons ici $W$ et $V^{[d]}$ comme des faisceaux sur le site fppf de $\operatorname{Spec}(k)$; nous n'aurons à utiliser aucun des résultats profonds de représentabilité connus, comme le fait que $V^{[d]}$ est un $k$-espace [Art69, §6] et est même quasi-projectif si $V$ l'est ([Gro60, §4], [Ber09, $\S 2]$ ou $\left[\right.$ Nit05, §5.5]). Le seul cas qui nous servira dans l'article est celui où $Q^{\prime}$ est fini sur $k^{\prime}$, pour lequel la représentabilité (et même la projectivité de $V^{[d]}$ ) est très facile : voir le lemme 2.5 .5 plus bas.

2.5.2 Rappelons que l'on a un morphisme de foncteurs

$$
u=u_{Q^{\prime} / k^{\prime} / k}: W \rightarrow V^{[d]}
$$

défini ainsi : si $S$ est un $k$-schéma, on associe à $w \in W(S)$ le $k^{\prime}$-morphisme $w^{\prime}: S \times_{k} k^{\prime} \rightarrow Q^{\prime}$. Son graphe

$$
\Gamma_{w^{\prime}} \subset Q^{\prime} \times_{k^{\prime}}\left(S \times_{k} k^{\prime}\right)=V \times_{k} S
$$

est un sous $S$-schéma fermé de $V \times_{k} S$, isomorphe à $S \times_{k} k^{\prime}$ et donc fini et libre de rang $d$ sur $S$ : c'est le point $u_{Q^{\prime} / k^{\prime} / k}(S)(w) \in V^{[d]}(S)$ voulu.

Lemme. (1) Pour tout $k$-schéma $S$, l'application $u_{Q^{\prime} / k^{\prime} / k}(S)$ ci-dessus induit une bijection de $W(S)$ sur l'ensemble des $Z \subset Q^{\prime} \times_{k} S$ tels que le $S$-morphisme $\varphi_{Z}$ défini en 2.5.1 soit un isomorphisme.

En particulier, le morphisme de foncteurs $u: W \rightarrow V^{[d]}$ est représentable par une immersion ouverte. 


\section{Ofer Gabber, Philippe Gille et Laurent Moret-Bailly}

(2) Si $k^{\prime}$ est un corps, $u$ induit une bijection de $W(k)$ sur $V^{[d]}(k)$.

Démonstration. Un sous-schéma $Z \subset Q^{\prime} \times_{k} S$ tel que $\varphi_{Z}$ est un isomorphisme définit une section de $Q^{\prime} \times_{k} S=V \times_{k} S \rightarrow k^{\prime} \times_{k} S$, c'est-à-dire un point de $W(S)=\left(\prod_{k^{\prime} / k} Q^{\prime}\right)(S)$. La partie (1) est alors immédiate et laissée au lecteur. L'assertion (2) en résulte : si $k^{\prime}$ est un corps et si $Z \subset V$ est fini de rang $d$ sur $k$, alors $\varphi_{Z}: Z \rightarrow \operatorname{Spec}\left(k^{\prime}\right)$ est nécessairement un isomorphisme.

2.5.2.1 Remarques. De façon imagée, on peut formuler (2) en disant que $V^{[d]}$, vu comme compactification partielle de $W$, n'a pas de point $k$-rationnel à l'infini. Le même argument montre d'ailleurs qu'il n'a pas de point $K$-rationnel à l'infini, dès que $K$ est une extension de $k$ telle que $K \otimes_{k} k^{\prime}$ soit un corps : le cas utile pour nous sera celui où $k^{\prime}$ (resp. $K$ ) est une extension radicielle (resp. séparable) de $k$.

Dans la suite, nous allons généraliser cette propriété en remplaçant les points par les orbites sous l'action d'un groupe algébrique.

2.5.3 Version équivariante : orbites à l'infini. On garde les notations de 2.5.1 et 2.5.2, et l'on se donne de plus un $k$-groupe algébrique $G$ opérant à gauche sur $V$ par $k^{\prime}$-automorphismes, c'est-à-dire que $\sigma: V \rightarrow \operatorname{Spec}\left(k^{\prime}\right)$ est $G$-invariant. (Il reviendrait au même de se donner une action du $k^{\prime}$-groupe $G_{k^{\prime}}$ sur $Q^{\prime}$; l'action de $G$ sera plus commode à utiliser).

On en déduit formellement des actions de $G$ (vu comme foncteur en groupes) sur les foncteurs $W$ et $V^{[d]}$, et l'immersion ouverte $u$ est équivariante pour ces actions.

2.5.4 Théorème. Avec les hypothèses et notations de 2.5.3, soient $K$ une extension de $k$ et $J \subset V_{K}^{[d]}$ une $K$-orbite pour l'action de $G_{K}$ (cf. 2.1.3).

Alors, si $K \otimes_{k} k^{\prime}$ est un corps, on a $J \subset W_{K}$.

Démonstration. En remplaçant $k^{\prime}$ par $K \otimes_{k} k^{\prime}, Q^{\prime}$ par $Q_{K}^{\prime}$, etc., on peut supposer que $K=k$ (et que $k^{\prime}$ est un corps). Alors $y: J \rightarrow V^{[d]}$ définit un sous-espace $Z$ de $Q^{\prime} \times_{k} J$, fini localement libre de rang $d$ sur $J$, et stable sous $G$ (opérant sur $J \times_{k} Q^{\prime}$ par l'action produit). Par 2.5.2, il s'agit de montrer que le $J$-morphisme

$$
\varphi_{Z}: Z \longrightarrow k^{\prime} \times_{k} J
$$

défini en 2.5.1 est un isomorphisme. Il est décrit par un morphisme $G$-équivariant

$$
\psi_{Z}: k^{\prime} \otimes_{k} \mathscr{O}_{J} \longrightarrow \mathscr{A}:=\operatorname{pr}_{2 *}\left(\mathscr{O}_{Z}\right)
$$

de $\mathscr{O}_{J}$-algèbres finies localement libres de rang $d$, linéarisées pour l'action de $G$ sur $J$. En particulier le conoyau $\mathscr{C}$ de $\psi_{Z}$ est un $\mathscr{O}_{J}$-module cohérent $G$-linéarisé. Considérons, pour chaque $s \in \mathbb{N}$, la strate de Fitting (de $\operatorname{rang} s) F_{s}(\mathscr{C}) \subset J$ : c'est un sous-schéma localement fermé de $J$, tel que la restriction de $\mathscr{C}$ à $F_{s}(\mathscr{C})$ soit localement libre de rang $s$, et universel pour cette propriété (cf. $[G W 10, \S 11.8]$ ) Puisque $\mathscr{C}$ est $G$-linéarisé, chaque $F_{s}(\mathscr{C})$ est stable par $G$; comme $G$ opère transitivement sur $J$, on a donc $F_{s}(\mathscr{C})=\emptyset$ sauf pour une valeur $r$ de $s$, pour laquelle $F_{r}(\mathscr{C})=J$. En d'autres termes, $\mathscr{C}$ est localement libre de rang constant $r$.

Il reste à voir que $r=0$. Puisque $\mathscr{C}$ est localement libre de rang $r$, l'image de $\psi_{Z}$ est une algèbre quotient de $k^{\prime} \otimes_{k} \mathscr{O}_{J}$, localement libre de rang $d-r$, et définit donc un sous-schéma $T \subset k^{\prime} \times_{k} J$, fini localement libre sur $J$ de $\operatorname{rang} d-r$ et $G$-invariant. Comme $G$ opère transitivement sur $J$ et trivialement sur $\operatorname{Spec}\left(k^{\prime}\right), T$ provient par descente d'un sous- $k$-schéma $T_{0}$ de rang $d-r$ de $\operatorname{Spec}\left(k^{\prime}\right)$. Puisque $k^{\prime}$ est un corps, on a soit $T_{0}=\emptyset$ et $r=d$, soit $T_{0}=\operatorname{Spec}\left(k^{\prime}\right)$ et $r=0 ;$ mais le premier cas est exclu car $\psi_{Z}$ est un morphisme d'algèbres non nulles, donc n'est pas nul. 


\section{FibRÉS PRINCIPAUX SUR LES CORPS VALUÉS HENSÉLIENS}

2.5.5 Lemme. Soient $Y$ un $k$-schéma fini, et $d \in \mathbb{N}$. Alors le foncteur $Y^{[d]}:=\operatorname{Hilb}_{Y / k}^{d}$ des sousschémas finis de longueur $d$ de $Y$ est représentable par un $k$-schéma projectif, muni d'un faisceau ample $\underline{\operatorname{Aut}}_{k}(Y)$-linéarisé.

Démonstration. On pose $Y=\operatorname{Spec}(A)$ où $A$ est une $k$-algèbre finie; on note $A_{\text {lin }}$ le $k$-espace vectoriel sous-jacent à $A$, et $G$ le $k$-groupe $\underline{A u t}_{k}(Y)$; c'est évidemment un sous-schéma en groupes fermé de $\operatorname{GL}\left(A_{\operatorname{lin}}\right)$, et en particulier un $k$-groupe algébrique affine.

La projectivité de $Y^{[d]}$ est un fait général [Ber09, prop 2.13], mais se voit facilement ici : en effet, pour tout $k$-schéma $S$, un point de $Y^{[d]}(S)$ est la même chose qu'une $\mathscr{O}_{S^{-}}$-algèbre quotient de $\mathscr{O}_{S} \otimes_{k} A$, localement libre de rang $d$ comme $\mathscr{O}_{S}$-module. Ainsi, $Y^{[d]}$ est de façon naturelle un sousschéma fermé de la grassmannienne $\mathrm{Gr}_{d}\left(A_{\text {lin }}\right)$ des quotients de rang $d$ de $A_{\text {lin }}$. De plus, l'action de $G$ sur $Y^{[d]}$ est induite par son action naturelle sur $\operatorname{Gr}_{d}\left(A_{\text {lin }}\right)$, laquelle se factorise par celle de $\operatorname{GL}_{k}\left(A_{\text {lin }}\right)$, de sorte que le faisceau ample canonique sur $\operatorname{Gr}_{d}\left(A_{\text {lin }}\right)$ (qui est $\operatorname{GL}_{k}\left(A_{\text {lin }}\right)$-linéarisé) induit un faisceau ample $G$-linéarisé sur $Y^{[d]}$.

\section{Corps topologiquement henséliens; le cas des torseurs sous un groupe lisse}

On désigne par $F$ un corps topologique séparé; on notera $\widehat{F}$ son complété (qui est une $F$ algèbre topologique, et est un corps si la topologie de $F$ est définie par une valuation).

\subsection{Variétés sur un corps topologique; corps topologiquement henséliens}

On note $\mathrm{VAR}_{F}$ la catégorie des $F$-variétés, TOP celle des espaces topologiques, ENS celle des ensembles.

On peut (voir [Wei62, app. III], [KS83, p. 256]) d'une manière et d'une seule, associer à toute $F$-variété $X$ (ou plus généralement à tout $F$-schéma localement de type fini) une topologie sur l'ensemble $X(F)$, de telle sorte que les conditions suivantes soient vérifiées (dans lesquelles $X$ et $Y$ désignent des $F$-variétés quelconques) :

(i) si $X=\mathbb{A}_{F}^{1}$, la bijection naturelle de $X(F)$ sur $F$ est un homéomorphisme;

(ii) si $f: X \rightarrow Y$ est un $F$-morphisme, l'application induite $f(F): X(F) \rightarrow Y(F)$ est continue; si de plus $f$ est une immersion ouverte (resp. fermée), alors $f(F)$ est un plongement topologique ouvert (resp. fermé) ;

(iii) la bijection naturelle de $\left(X \times_{F} Y\right)(F)$ sur $X(F) \times Y(F)$ est un homéomorphisme;

(iv) si $X$ est un schéma séparé, alors $X(F)$ est un espace séparé.

(Ces conditions ne sont pas indépendantes : par exemple, la condition (iv) est conséquence des conditions (ii) et (iii) appliquées au morphisme diagonal $X \rightarrow X \times_{F} X$.)

On obtient ainsi un foncteur, noté $X \mapsto X(F)_{\text {top }}$ (ou encore $X \mapsto X_{\text {top }}$ ), de $\mathrm{VAR}_{F}$ dans TOP. Il résulte facilement des propriétés ci-dessus que ce foncteur transforme les immersions en plongements topologiques et commute aux produits fibrés.

3.1.1 Définition. Un corps topologique séparé $F$ est topologiquement hensélien si pour tout morphisme étale $f: X \rightarrow Y$ de $F$-variétés, l'application induite $f_{\text {top }}$ est un homéomorphisme local.

3.1.1.1 Remarques. Si l'on se limite aux topologies déduites de valuations ou de valeurs absolues (《V-topologies »), les corps topologiquement henséliens sont appelés t-henséliens dans l'article [PZ78], qui en donne diverses caractérisations. 


\section{Ofer Gabber, Philippe Gille et Laurent Moret-Bailly}

Outre les corps valués henséliens (proposition 3.1.4 plus bas), les corps $\mathbb{R}$ et $\mathbb{C}$ sont topologiquement henséliens (pour leur topologie classique), de même que leurs sous-corps $\overline{\mathbb{Q}}$ et $\overline{\mathbb{Q}} \cap \mathbb{R}$ et que tout corps réel clos muni de la topologie de l'ordre.

3.1.2 Lemme. Soit $F$ un corps topologiquement hensélien, et soit $f: Y \rightarrow X$ un morphisme lisse de $F$-variétés.

Alors, pour tout $y \in Y(F)$, il existe un voisinage ouvert $\Omega$ de $f(y)$ dans $X_{\text {top }}$ tel que l'application induite $f_{\text {top }}: Y_{\text {top }} \rightarrow X_{\text {top }}$ admette une section continue $\Omega \rightarrow Y_{\text {top }}$.

En particulier, $f_{\text {top }}$ est ouverte.

Démonstration. Remarquer que si $y \in Y(F)$, il existe un morphisme $h: Z \rightarrow Y$ et un point $z \in Z(F)$ tels que $h(z)=y$ et que le composé $f \circ h: Z \rightarrow X$ soit étale [BLR90, $\S 2.2$, proposition 14].

3.1.3 Remarque. Réciproquement, soit $F$ un corps topologique séparé tel que $f_{\text {top }}$ soit ouverte pour tout morphisme lisse $f: X \rightarrow Y$ de $F$-variétés. Alors $F$ est topologiquement hensélien : le lecteur le vérifiera en utilisant le fait que si $f$ est étale, le morphisme diagonal $X \rightarrow X \times_{Y} X$ est une immersion ouverte. (Cette remarque n'est pas utilisée dans la suite).

Nous allons justifier cette terminologie par l'énoncé suivant (sans doute bien connu, mais difficile à trouver sous cette forme dans la littérature).

\subsubsection{Proposition. Tout corps valué hensélien est topologiquement hensélien.}

Démonstration. Soient $F$ un corps valué hensélien et $f: X \rightarrow Y$ un morphisme étale de $F$ variétés. Soit $x \in X(F)$ et soit $y$ son image dans $Y(F)$ : montrons que $f_{\text {top }}$ est un homéomorphisme local au point $x$. Bien entendu, il nous suffira pour cela de supposer que $f$ est étale $a u$ point $x$, ce que nous ferons systématiquement dans les réductions qui vont suivre.

On peut supposer que $Y=\operatorname{Spec}(A)$ est affine et que $X$ est un ouvert de $\operatorname{Spec}(A[T] / P(T))$ où $P \in A[T]$ est un polynôme (unitaire, si l'on veut) tel que $P^{\prime}(x) \neq 0$ [BLR90, $\S 2.3$, proposition 3]. Sans perte de généralité, on peut supposer que $X=\operatorname{Spec}(A[T] / P(T))$. On plonge $Y$ dans un espace affine $\widetilde{Y}=\mathbb{A}_{F}^{n}=\operatorname{Spec}(\widetilde{A})$ et on relève $P(T)$ en un polynôme unitaire $\widetilde{P}(T) \in \widetilde{A}[T]$. Remplaçant $Y$ par $\widetilde{Y}$ et $P$ par $\widetilde{P}$, nous sommes ramenés à la situation où $Y=\mathbb{A}_{F}^{n}=$ Spec $F[\underline{Z}]=$ $\operatorname{Spec} F\left[Z_{1}, \ldots, Z_{n}\right], X=\operatorname{Spec}(F[\underline{Z}, T] /(P))$ où $P$ est unitaire en $T$, et où $y$ (resp. $x$ ) est l'origine de $\mathbb{A}_{F}^{n}$ (resp. de $\mathbb{A}_{F}^{n+1}$ ). En outre, la projection $X \rightarrow Y$ étant étale en $x$, nous pouvons choisir les coordonnées de manière que l'hyperplan tangent en $x$ à $X$ ait pour équation $T=0$, de sorte que $P$ est (à un scalaire inversible près) de la forme

$$
P(\underline{Z}, T)=T+\sum_{|I|+j \geqslant 2} a_{I, j} \underline{Z}^{I} T^{j} \quad\left(a_{I, j} \in F\right) .
$$

Notons $R$ l'anneau de la valuation $v$, et $\mathfrak{m}$ son idéal maximal. Pour $\alpha \in F^{\times}$, nous pouvons remplacer $P$ par $P_{\alpha}(\underline{Z}, T):=\frac{1}{\alpha} P(\alpha \underline{Z}, \alpha T)$. Le coefficient de $\underline{Z}^{I} T^{j}$ dans $P_{\alpha}$ est $\alpha^{|I|+j-1} a_{I, j}$ : dans cette formule l'exposant de $\alpha$ est strictement positif donc, prenant $\alpha$ assez proche de 0 , on peut supposer que les coefficients $a_{I, j}$ sont dans $\mathfrak{m}$. La propriété de Hensel montre alors que pour chaque $\underline{z} \in R^{n}$, le polynôme $P(\underline{z}, T) \in R[T]$ admet une unique racine $t(\underline{z})$ dans $\mathfrak{m}$. En d'autres termes, $f_{\text {top }}$ induit une bijection entre $f_{\text {top }}^{-1}\left(R^{n}\right) \cap\left(R^{n} \times \mathfrak{m}\right)$ (voisinage de $x$ dans $X_{\text {top }}$ ) et $R^{n}$ (voisinage de $y$ dans $F^{n}$ ).

Il reste à voir que l'application $\underline{z} \mapsto t(\underline{z})$ est continue à l'origine, et pour cela il suffit d'établir que

$$
v(t(\underline{z})) \geqslant \min _{i=1, \ldots, n} v\left(z_{i}\right)
$$




\section{FibRÉS PRINCIPAUX SUR LES CORPS VALUÉS HENSÉLIENS}

En d'autres termes, nous devons montrer que si $\underline{z}=\left(z_{1}, \ldots, z_{n}\right) \in R^{n}$ et $t \in \mathfrak{m}$ vérifient

$$
v(t)<\min _{i=1, \ldots, n} v\left(z_{i}\right)
$$

(ce qui implique que $t \neq 0$ ), alors on a $P(\underline{z}, t) \neq 0$. Il suffit pour cela de voir que chaque terme $\underline{z}^{I} t^{j}$, pour $|I|+j \geqslant 2$, est de valuation strictement supérieure à $v(t)$ (rappelons que $\left.v\left(a_{I, j}\right)>0\right)$. Or c'est vrai si $|I| \geqslant 1$ vu l'hypothèse sur les $v\left(z_{i}\right)$, et c'est vrai si $I=0$ car alors $j \geqslant 2$ et $t \in \mathfrak{m} \backslash\{0\}$.

\subsection{Extension aux espaces algébriques}

À partir de 3.2.6, le corps topologique $F$ sera toujours supposé topologiquement hensélien.

On identifie la catégorie $\mathrm{VAR}_{F}$ à une sous-catégorie pleine de la catégorie $\mathrm{ALG}_{F}$ des $F$-espaces (c'est-à-dire, rappelons-le, des $F$-espaces algébriques quasi-séparés de type fini). On se propose de définir, pour tout $F$-espace $X$, une topologie sur $X(F)$, qui coïncide avec celle déjà définie lorsque $X$ est un schéma. Le cas d'un corps valué complet de rang 1 est traité dans [Con12, §5]. Pour ne pas allonger démesurément ce travail, nous omettons ici la plupart des démonstrations.

3.2.1 Définition. Soit $X$ un $F$-espace. On munit $X(F)$ de la topologie suivante : une partie $\Omega \subset X(F)$ est ouverte si et seulement si, pour toute $F$-variété $Z$ et tout $F$-morphisme $\varphi: Z \rightarrow X$, l'ensemble $\varphi^{-1}(\Omega) \subset Z(F)$ est un ouvert de $Z_{\text {top }}$.

L'espace topologique ainsi obtenu sera noté $X_{\text {top }}$ ou $X(F)_{\text {top }}$.

3.2.2 Remarque. En d'autres termes, on munit $X(F)$ de la topologie la plus fine rendant continues toutes les applications $\varphi(F)$ lorsque $\varphi$ parcourt tous les $F$-morphismes d'une $F$-variété vers $X$. Noter qu'il est clair que l'on peut se limiter aux variétés $Z$ qui sont affines.

\subsubsection{Proposition.}

(1) Si $X$ est une $F$-variété, la topologie définie en 3.2.1 (où $X$ est vue comme $F$-espace) coïncide avec celle déjà définie en 3.1 .

(2) Soit $f: X \rightarrow Y$ un morphisme de F-espaces. Alors :

(i) l'application $f_{\text {top }}: X_{\text {top }} \rightarrow Y_{\text {top }}$ induite par $f$ sur les points $F$-rationnels est continue;

(ii) si $f$ est une immersion (resp. une immersion ouverte, resp. une immersion fermée), alors $f_{\text {top }}$ est un homéomorphisme sur son image, qui est localement fermée (resp. ouverte, resp. fermée) dans $Y_{\text {top }}$.

Démonstration. Les assertions (1) et (2) (i) sont immédiates à partir de la définition 3.2.1. Montrons l'assertion (2) (ii) : si $f$ est une immersion alors $f_{\text {top }}$ est injective, donc il suffit de voir que si $f$ est une immersion ouverte (resp. fermée) alors $f_{\text {top }}$ est une application ouverte (resp. fermée), le cas d'une immersion arbitraire en résultant par composition.

Traitons le cas d'une immersion ouverte (celui d'une immersion fermée est entièrement analogue). Soit $\Omega$ un ouvert de $X_{\text {top }}$, et montrons que $f_{\text {top }}(\Omega)$ est ouvert dans $Y_{\text {top }}$ : soit donc $h: Y^{\prime} \rightarrow Y$ un $F$-morphisme, où $Y^{\prime}$ est une variété. Il s'agit de voir que $h_{\text {top }}^{-1}\left(f_{\text {top }}(\Omega)\right)$ est un ouvert de $Y_{\text {top }}^{\prime}$ Posons $X^{\prime}=X \times_{Y} Y^{\prime}$ et soient $f^{\prime}: X^{\prime} \rightarrow Y^{\prime}$ et $h^{\prime}: X^{\prime} \rightarrow X$ les morphismes évidents. Alors $f^{\prime}$ est une immersion ouverte, et en particulier $X^{\prime}$ est un schéma. De plus $h_{\text {top }}^{-1}\left(f_{\text {top }}(\Omega)\right)=f_{\text {top }}^{\prime}\left(h_{\text {top }}^{\prime-1}(\Omega)\right)$ qui est bien ouvert dans $Y_{\text {top }}^{\prime}$ puisque $h_{\text {top }}^{\prime-1}(\Omega)$ est ouvert dans $X_{\text {top }}^{\prime}$ et que $f^{\prime}$ est une immersion ouverte entre variétés.

Lorsque $F$ est topologiquement hensélien, on dispose d'une autre caractérisation de la topologie de 3.2.1, en termes de recouvrements étales (cf. 3.2.6 plus bas). Pour y parvenir, nous aurons 


\section{Ofer Gabber, Philippe Gille et Laurent Moret-Bailly}

besoin de l'énoncé suivant, variante d'un résultat de Gruson et Raynaud [GR71, I, proposition 5.7.6], et de son corollaire :

3.2.4 Proposition. [CLO09, Theorem 3.1.1] Soit $X$ un espace algébrique quasi-compact et quasi-séparé. Il existe une suite croissante finie

$$
\emptyset=U_{0} \subset U_{1} \subset \cdots \subset U_{r}=X
$$

d'ouverts quasi-compacts de $X$, avec la propriété suivante : pour chaque $i>0$, si l'on note $Z_{i}$ le sous-espace fermé réduit de $U_{i}$ complémentaire de $U_{i-1}$, il existe un morphisme étale surjectif $\pi_{i}: Y_{i} \rightarrow U_{i}$ qui est un isomorphisme au-dessus de $Z_{i}$, et où $Y_{i}$ est un schéma quasi-compact et séparé.

3.2.5 Corollaire. Soit $X$ un espace algébrique quasi-compact et quasi-séparé. Il existe un schéma affine $Y$ et un morphisme étale surjectif $\pi: Y \rightarrow X$ tel que pour tout anneau artinien $A$ l'application $Y(A) \rightarrow X(A)$ induite par $\pi$ soit surjective.

3.2.6 Proposition. On suppose $F$ topologiquement hensélien.

Soit $Y$ un $F$-espace. Fixons un $F$-morphisme étale $\pi: X \rightarrow Y$, où $X$ est une $F$-variété séparée. Alors $\pi_{\text {top }}: X_{\text {top }} \rightarrow Y_{\text {top }}$ est ouverte.

En particulier, si de plus $\pi$ induit une surjection de $X(F)$ sur $Y(F)$ (étant donné $Y$, un tel morphisme $\pi$ existe, d'après 3.2.5), alors $Y_{\text {top }}$ s'identifie au quotient de $X_{\text {top }}$ par la relation d'équivalence définie par $\pi_{\text {top }}$.

3.2.7 Produits fibrés. Considérons un diagramme cartésien

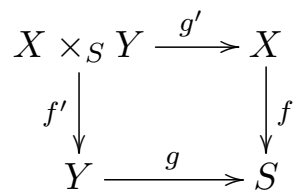

de $F$-espaces. Notons $j: X \times_{S} Y \rightarrow X \times_{F} Y$ le monomorphisme naturel : il induit une injection continue

$$
j_{\text {top }}:\left(X \times_{S} Y\right)_{\text {top }} \hookrightarrow\left(X \times_{F} Y\right)_{\text {top }} .
$$

On a d'autre part une application continue naturelle

$$
\alpha:\left(X \times_{S} Y\right)_{\text {top }} \rightarrow X_{\text {top }} \times_{S_{\text {top }}} Y_{\text {top }}
$$

déduite des propriétés universelles, et évidemment bijective.

Proposition. Avec les hypothèses ci-dessus, on suppose $F$ topologiquement hensélien.

(1) Les conditions suivantes sont équivalentes :

(i) $\alpha$ est un homéomorphisme;

(ii) $j_{\text {top }}$ est un homéomorphisme sur son image.

(2) Les conditions de (1) sont satisfaites dans les deux cas suivants :

(iii) l'un des morphismes $f, f^{\prime}, g, g^{\prime}$ est une immersion;

(iv) $S$ est localement séparé.

3.2.7.1 Remarques. (1) Bien entendu, un cas particulier de (iv) est celui où $S=\operatorname{Spec}(F)$. En d'autres termes, le foncteur $X \mapsto X_{\text {top }}$ commute aux produits de $F$-espaces. 


\section{FibRÉS PRINCIPAUX SUR LES CORPS VALUÉS HENSÉLIENS}

(2) Le cas (iii) n'utilise pas l'hypothèse que $F$ est topologiquement hensélien.

(3) Un cas particulier utile de (iii) est le fait que pour $f: X \rightarrow S$ quelconque et $s \in S(F)$, la fibre $f_{\text {top }}^{-1}(s)$ s'identifie à $\left(f^{-1}(s)\right)_{\text {top }}$, l'inclusion d'un point rationnel étant toujours une immersion.

(4) On verra en 7.3 un exemple où les conditions de (1) ne sont pas satisfaites.

3.2.8 Proposition. (morphismes lisses et étales) On suppose $F$ topologiquement hensélien. Soit $f: X \rightarrow Y$ un morphisme lisse de $F$-espaces. Alors :

(1) $f_{\text {top }}: X_{\text {top }} \rightarrow Y_{\text {top }}$ est ouverte.

(2) On suppose que $f$ est étale et que, de plus, la bijection naturelle $\left(X \times_{Y} X\right)_{\text {top }} \rightarrow X_{\text {top }} \times_{Y_{\text {top }}} X_{\text {top }}$ est un homéomorphisme (condition vérifiée notamment, d'après 3.2.7, si $Y$ est localement séparé). Alors $f_{\text {top }}$ est un homéomorphisme local.

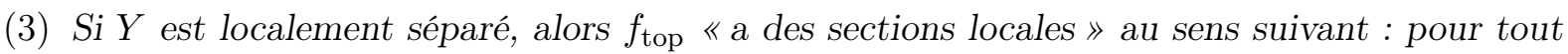
$x \in X_{\text {top }}$, il existe un voisinage $V$ de $f(x)$ dans $Y_{\text {top }}$ et une section continue $s: V \rightarrow X_{\text {top }}$ de $f$ sur $V$ telle que $s(f(x))=x$.

\subsection{Corps valués henséliens : utilisation de modèles entiers}

On suppose dans cette section que $F$ est un corps muni d'une valuation (non triviale) $v$ d'anneau $A$, et de la topologie associée. On notera $\mathfrak{I}$ l'ensemble des idéaux stricts et non nuls de $A, \mathfrak{m} \in \mathfrak{I}$ son idéal maximal, $k=A / \mathfrak{m}$ son corps résiduel.

On se propose de décrire l'espace $X_{\text {top }}$, où $X$ est une $F$-variété, en termes des «modèles entiers 》 de $X$, c'est-à-dire des $A$-espaces algébriques $\mathscr{X}$ de type fini munis d'un isomorphisme $\mathscr{X}_{F} \stackrel{\sim}{\rightarrow} X$. Comme dans la section 3.2 , les démonstrations sont omises.

3.3.1 Topologie sur les points entiers. Soit $\mathscr{X}$ un $A$-espace algébrique quasi-séparé de type fini. (Le lecteur pourra constater que les constructions qui suivent ont un sens pour des foncteurs plus généraux).

On notera

$$
\begin{aligned}
& \rho_{\mathscr{X}, J}: \mathscr{X}(A) \longrightarrow \mathscr{X}(A / J) \quad(J \in \mathfrak{I}) \\
& \rho_{\mathscr{X}}: \mathscr{X}(A) \longrightarrow \lim _{J \in \mathfrak{I}} \mathscr{X}(A / J)
\end{aligned}
$$

les applications évidentes; on utilisera aussi la notation « $x \bmod J » \operatorname{pour} \rho_{\mathscr{X}, J}(x)$.

On munira chaque $\mathscr{X}(A / J)$ de la topologie discrète, $\varliminf_{J \in \mathfrak{I}} \mathscr{X}(A / J)$ de la topologie limite projective, et l'ensemble $\mathscr{X}(A)$ de la topologie induite via $\rho_{\mathscr{X}}$. L'espace topologique obtenu sera noté $\mathscr{X}(A)_{A, \text { top }}$, ou $\mathscr{X}_{A, \text { top }}$.

3.3.1.1 Proposition. L'application $\rho_{\mathscr{X}}$ est injective. En particulier, l'espace $\mathscr{X}_{A \text {,top }}$ est séparé.

Démonstration. Soient $x$ et $x^{\prime}$ dans $\mathscr{X}(A)$ ayant même image par $\rho_{\mathscr{X}}$. Le noyau de la double flèche

$$
\operatorname{Spec}(A) \underset{x^{\prime}}{\stackrel{x}{\rightrightarrows}} \mathscr{X}
$$

est alors représenté par un monomorphisme de $A$-schémas $Z \rightarrow \operatorname{Spec}(A)$; d'autre part, le fait que $\rho_{\mathscr{X}}(x)=\rho_{\mathscr{X}}\left(x^{\prime}\right)$ entraîne l'existence, pour chaque $J \in \mathfrak{I}$, d'un $A$-morphisme $s_{J}: \operatorname{Spec}(A / J) \rightarrow$ $Z$. Comme $Z$ est un schéma (et les $s_{J}$ automatiquement compatibles), on en déduit un $A$ morphisme $\operatorname{Spec}(\widehat{A}) \rightarrow Z$. Ainsi, $Z \rightarrow \operatorname{Spec}(A)$ est à la fois un monomorphisme de schémas et un épimorphisme de faisceaux fpqc (rappelons que $\widehat{A}$ est fidèlement plat sur $A$ ) ; c'est donc un isomorphisme, de sorte que $x=x^{\prime}$. 


\section{Ofer Gabber, Philippe Gille et Laurent Moret-Bailly}

Si $x \in \mathscr{X}(A)$ et $J \in \mathfrak{I}$, on notera $B_{\mathscr{X}}(x, J)$ la « boule de rayon $J »$

$$
B_{\mathscr{X}}(x, J)=\rho_{X, J}^{-1}\left(\rho_{X, J}(x)\right)=\left\{x^{\prime} \in \mathscr{X}(A) \mid x^{\prime} \bmod J=x \bmod J\right\}
$$

(de sorte que la famille $\left(B_{\mathscr{X}}(x, J)\right)_{J \in \mathfrak{I}}$ est une base de voisinages ouverts de $x$ dans $\mathscr{X}_{A \text {,top }}$ ).

Si $\varphi: \mathscr{X} \rightarrow \mathscr{Y}$ est un morphisme d'espaces algébriques quasi-séparés de type fini sur $A$, l'application naturelle $\varphi_{A \text {,top }}: \mathscr{X}_{A \text {,top }} \rightarrow \mathscr{Y}_{A \text {,top }}$ est continue, et plus précisément envoie $B_{\mathscr{X}}(x, J)$ dans $B_{\mathscr{Y}}(\varphi(x), J)$ pour tout $x \in \mathscr{X}(A)$ et tout $J \in \mathfrak{I}$.

3.3.2 Proposition. On garde les notations de 3.3.1; en particulier les lettres $\mathscr{X}$, $\mathscr{Y}$ désignent des $A$-espaces algébriques quasi-séparés de type fini, et $\varphi: \mathscr{X} \rightarrow \mathscr{Y}$ un A-morphisme.

(1) Le foncteur $\mathscr{X} \mapsto \mathscr{X}_{A \text {,top }}$ (de la catégorie des $A$-espaces algébriques quasi-séparés de type fini dans celle des espaces topologiques) commute aux produits fibrés finis.

(2) Si $\varphi$ est un monomorphisme, alors $\varphi_{A \text {,top }}$ est un plongement topologique fermé.

(3) Supposons $A$ hensélien et $\varphi$ étale. Alors $\varphi_{A \text {,top }}$ est un homéomorphisme local; plus précisément, pour tout $x \in \mathscr{X}(A)$ et tout $J \in \mathfrak{I}, \varphi_{A \text {,top }}$ induit un homéomorphisme de $B_{\mathscr{X}}(x, J)$ sur $B_{\mathscr{Y}}(f(x), J)$.

3.3.2.1 Remarque (non utilisée dans la suite). Avec les notations de 3.3.1, il est clair que l'application $\rho_{\mathscr{X}}$ se factorise par $\mathscr{X}(\widehat{A})$, puisque $A / J \stackrel{\sim}{\rightarrow} \widehat{A} / J \widehat{A}$ pour tout $J \in \mathfrak{I}$. En fait, on voit facilement que l'ensemble $\lim _{J \in \mathfrak{I}} \mathscr{X}(A / J)$ s'identifie à $\mathscr{X}(\widehat{A})$ si $\widehat{A}$ est hensélien, ou de façon équivalente si chacun des quotients $A / J(J \in \mathfrak{I})$ est hensélien; ce sera le cas notamment si $A$ est hensélien, ou si $\Gamma$ est de rang 1 (c'est-à-dire si $\operatorname{dim} A=1$ ).

3.3.3 Comparaison des topologies sur les $A$-points et les $F$-points. Gardons les hypothèses et notations de 3.3.1; en outre, notons $j: \operatorname{Spec}(F) \hookrightarrow \operatorname{Spec}(A)$ le morphisme canonique, et considérons l'application naturelle

$$
\begin{aligned}
j^{*}: \mathscr{X}_{A, \mathrm{top}} & \longrightarrow \mathscr{X}_{F, \text { top }} \\
x & \longmapsto x \circ j .
\end{aligned}
$$

Rappelons que $\mathscr{X}_{A \text {,top }}$ est l'ensemble $\mathscr{X}(A)$ muni de la topologie de 3.3 .1 ; par ailleurs, $\mathscr{X}_{F, \text { top }}$ désigne l'ensemble $\mathscr{X}(F)$, identifié à $\mathscr{X}_{F}(F)$ et muni à ce titre de la topologie du $\S 3.2$ (puisque $\mathscr{X}_{F}=\mathscr{X} \otimes_{A} F$ est un $F$-espace algébrique de type fini).

3.3.4 Proposition. Avec les notations de 3.3.3, on suppose en outre que $A$ est hensélien, ou bien que $\mathscr{X}$ est un schéma. Alors :

(1) $j^{*}$ est continue et ouverte.

(2) Si $\mathscr{X}_{F}$ est localement séparé, $j^{*}$ est un homéomorphisme local.

(3) Si $\mathscr{X}$ est localement séparé, alors, pour tout $x \in \mathscr{X}(A), j^{*}$ induit un homéomorphisme de $B_{\mathscr{X}}(x, \mathfrak{m})$ sur un ouvert de $\mathscr{X}(F)$.

(4) Si $\mathscr{X}$ est séparé (resp. propre sur A), alors $j^{*}$ est un plongement topologique ouvert (resp. un homéomorphisme).

Donnons quelques indications sur la preuve de 3.3.4.

(a) On commence par montrer que si $\mathscr{X}$ est localement séparé, alors (sans hypothèse hensélienne sur $A$ ) la restriction de $j^{*}$ à $B_{\mathscr{X}}(x, \mathfrak{m})$ est injective (pour $y$ et $y^{\prime}$ ayant même image, considérer le noyau de $\left.\operatorname{Spec}(A) \underset{y}{\stackrel{y^{\prime}}{\rightrightarrows}} \mathscr{X}\right)$. 


\section{FIBRÉS PRINCIPAUX SUR LES CORPS VALUÉS HENSÉLIENS}

(b) Il en résulte que pour $\mathscr{X}$ localement séparé, les assertions (2) et (3) sont équivalentes.

(c) On montre l'assertion (3) lorsque $\mathscr{X}$ est un schéma par réduction au cas affine.

(d) Pour $A$ hensélien et $\mathscr{X}_{F}$ localement séparé, il y a un $A$-schéma affine $\mathscr{X}^{\prime}$ et un $A$-morphisme étale $\pi: \mathscr{X}^{\prime} \rightarrow \mathscr{X}$ tels que $x$ se relève en $x^{\prime} \in \mathscr{X}^{\prime}(A)$. On montre alors l'assertion (2) en utilisant 3.3.2 (3) (appliqué à $\pi$ ), 3.2.8 (2) (appliqué à $\pi_{F}$ ), et l'assertion (3) pour les schémas (appliquée à $\left.\mathscr{X}^{\prime}\right)$.

(e) L'assertion (4) est conséquence évidente de l'assertion (2) et du fait que, pour $\mathscr{X}$ séparé (reps. propre), $j^{*}$ est injective (resp. bijective).

(f) Enfin, l'assertion (1) est immédiate (et résulte d'ailleurs de (2)) si $\mathscr{X}$ est un schéma affine; pour $A$ hensélien, on se ramène à ce cas en utilisant 3.3.2 (3) comme en (d).

\subsection{Torseurs sous un groupe lisse}

3.4.1 Proposition. Soit $F$ un corps topologiquement hensélien. Soient $G$ un $F$-groupe algébrique lisse, $Y$ un $F$-espace et $f: X \rightarrow Y$ un $G$-torseur au-dessus de $Y$ (de sorte que $X$ est aussi un F-espace).

Alors $f_{\text {top }}: X_{\text {top }} \rightarrow Y_{\text {top }}$ est ouverte, et son image $I \subset Y_{\text {top }}$ est ouverte et fermée.

Si de plus $Y$ est localement séparé, l'application induite $X_{\text {top }} \rightarrow I$ est une $G_{\text {top }}$-fibration principale.

Démonstration. Comme $f$ est lisse, $f_{\text {top }}$ est ouverte $(3.2 .8(1))$, et son image est ouverte. Cette image est l'ensemble des $y \in Y(F)$ tels que le $G$-torseur $X_{y}:=f^{-1}(y) \operatorname{sur} \operatorname{Spec}(F)$ soit trivial. Elle est donc fermée en vertu du lemme 3.4.1.1 ci-dessous.

La dernière assertion vient du fait que $X_{\text {top }} \rightarrow Y_{\text {top }}$ est automatiquement un pseudo-torseur sous $G_{\text {top }}$ par fonctorialité (c'est-à-dire que l'application $G_{\text {top }} \times X_{\text {top }} \rightarrow X_{\text {top }} \times_{Y_{\text {top }}} X_{\text {top }}$ donnée par $(g, x) \mapsto(g x, x)$ est un homéomorphisme; on utilise ici la compatibilité aux produits fibrés), et que $f_{\text {top }}$ admet des sections locales au voisinage de tout point de $I(3.2 .8(3))$.

\subsubsection{Lemme. Sous les hypothèses de la proposition 3.4.1, l'application}

$$
\begin{aligned}
{[f]: Y_{\mathrm{top}} } & \longrightarrow \mathrm{H}_{\mathrm{et}}^{1}(F, G) \\
y & \longmapsto\left[X_{y}\right]
\end{aligned}
$$

est localement constante.

Démonstration. Fixons une classe $\xi \in \mathrm{H}_{\mathrm{et}}^{1}(F, G)$, et soit $T$ un $G$-torseur sur $\operatorname{Spec}(F)$ de classe $\xi$. Considérons le $G$-torseur « constant» $T_{Y}=T \times_{F} Y$ au-dessus de $Y$. Le faisceau étale $I:=$ $\underline{\operatorname{Isom}}_{G_{Y}}\left(T_{Y}, X\right)$ des morphismes (de $G$-torseurs sur $Y$ ) de $T_{Y}$ vers $X$ est un torseur sous le groupe des $G$-automorphismes de $T$; ce groupe est une forme intérieure de $G$ et donc un $F$-groupe lisse, de sorte que l'image de $I(F)$ dans $Y_{\text {top }}$ est ouverte. Or celle-ci n'est autre que $[f]^{-1}(\xi)$, d'où la conclusion.

3.4.2 Proposition. Soit $F$ un corps topologiquement hensélien. Soient $G$ un $F$-groupe algébrique, $Y$ un $F$-espace, et $f: X \rightarrow Y$ un $G$-torseur au-dessus de $Y$.

On note $I \subset Y_{\text {top }}$ l'image de $f_{\text {top }}$. Considérons les propriétés suivantes :

(i) l'application induite $X_{\text {top }} \rightarrow I$ est une $G_{\text {top }}$-fibration principale;

(ii) l'application induite $X_{\text {top }} \rightarrow I$ est ouverte;

(iii) l'application induite $X_{\text {top }} / G(F) \rightarrow I$ est un homéomorphisme; 


\section{Ofer Gabber, Philippe Gille et Laurent Moret-Bailly}

(iv) $f_{\text {top }}: X_{\text {top }} \rightarrow Y_{\text {top }}$ est stricte (1.7).

Alors on a les implications (i) $\Rightarrow($ ii $) \Leftrightarrow($ iii $) \Leftrightarrow($ iv), et les quatre conditions sont équivalentes si $Y$ est localement séparé.

3.4.2.1 Remarque. Lorsque $F$ est un corps valué admissible, cette proposition est une conséquence triviale du théorème principal 1.2 : les propriétés (ii) à (iv) sont vraies, et la propriété (i) l'est si $Y$ est localement séparé. Comme le théorème 1.2 sera démontré sans utiliser 3.4.2, cette proposition est donc redondante dans ce cas. Ceci étant, elle donne un résultat partiel dans la direction de 1.2 sans faire intervenir les compactifications.

Démonstration de la proposition. Les implications (i) $\Rightarrow($ ii $) \Rightarrow($ iii $) \Leftrightarrow($ iv) sont immédiates; en outre (iii) $\Rightarrow$ (ii) vient du fait que la relation d'équivalence déduite d'une action continue de groupe est toujours ouverte.

Supposons maintenant la propriété (ii) vérifiée et $Y$ localement séparé, et montrons la propriété (i). Posons $X^{\prime}=X / G^{\natural}$, où $G^{\natural}$ est défini en 2.4 ; noter en particulier que $G_{\text {top }}^{\natural}=G_{\text {top }}$. Alors $f$ se factorise suivant

$$
f: X \stackrel{f^{\prime}}{\longrightarrow} X^{\prime} \stackrel{g}{\longrightarrow} Y .
$$

Notons $I^{\prime} \subset X_{\text {top }}^{\prime}$ l'image de $f_{\text {top }}^{\prime}$. On a un diagramme d'espaces topologiques

$$
X_{\text {top }} \stackrel{f_{1}^{\prime}}{\longrightarrow} I^{\prime} \stackrel{g_{1}}{\longrightarrow} I \hookrightarrow Y_{\text {top }}
$$

où $f_{1}^{\prime}$ est surjective par définition de $I^{\prime}$, et $g_{1}$ bijective par 2.4.2. Par hypothèse la composée $g_{1} \circ f_{1}^{\prime}$ est ouverte. On en déduit que $g_{1}$ est ouverte, donc est un homéomorphisme de $I^{\prime}$ sur $I$. Il suffit donc de voir que $f_{1}^{\prime}$ est une $G_{\text {top }}^{\natural}$-fibration principale. Comme $f^{\prime}$ est un $G^{\natural}$-torseur et que $G^{\natural}$ est lisse, il suffit pour cela d'appliquer 3.4.1 en remarquant que $X^{\prime}$ est localement séparé : cette dernière propriété résulte de l'hypothèse sur $Y$ et du fait que $g: X^{\prime} \rightarrow Y$ est un morphisme séparé (il est localement isomorphe, pour la topologie fppf sur sur $Y$, à la projection $\left.Y \times\left(G / G^{\natural}\right) \rightarrow Y\right)$.

\subsection{Corps valués henséliens : approximation faible et applications}

\subsubsection{Rappels sur les corps valués henséliens.}

Soit $(K, v)$ un corps valué, d'anneau de valuation $A$. Rappelons que pour que $(K, v)$ soit hensélien (c'est-à-dire pour que $A$ soit un anneau local hensélien) il faut et il suffit que pour toute extension finie $L$ de $K$, la valuation $v$ se prolonge de manière unique en une valuation de $L$ [War89, théorème 32.8]. Dans ce cas, on supposera toujours $L$ muni de cette valuation, et de la topologie associée.

On prendra garde qu'un corps valué complet n'est pas nécessairement hensélien ; c'est toutefois vrai si la valuation est de rang 1.

Supposons $(K, v)$ hensélien; notons $\widehat{K}$ son complété, et $K_{s}$ une clôture séparable de $K$. Alors $\widehat{K}$ est hensélien (c'est immédiat) et de plus $K$ est séparablement fermé dans $\widehat{K}$ [War89, théorème 32.19], de sorte que $\widehat{K} \otimes_{K} K_{s}$ est un corps. Le corps $\widehat{K} \otimes_{K} K_{s}$ est même une clôture séparable de $\widehat{K}$. En effet, en vertu du théorème de l'élément primitif, une extension finie séparable de $\widehat{K}$ est isomorphe à $\widehat{K}[t] / P(t)$ où $P$ désigne un $\widehat{K}$-polynôme unitaire séparable; si $Q$ est un $K$-polynôme unitaire (séparable) assez proche de $P$, alors $P(T)$ et $Q(T)$ ont même corps de décomposition sur $\widehat{K}$ [War89, 32.20], donc $P$ se décompose sur $\widehat{K} \otimes_{K} K_{s}$. On a montré que $\widehat{K} \otimes_{K} K_{s}$ est une clôture séparable de $\widehat{K}$, on a donc un isomorphisme $\operatorname{Gal}\left(\widehat{K} \otimes_{K} K_{s} / \widehat{K}\right) \stackrel{\sim}{\longrightarrow} \operatorname{Gal}\left(K_{s} / K\right)$.

3.5.2 Proposition. (approximation faible) Soient $F$ un corps valué hensélien et $X$ un $F$-espace. On suppose que $X$ est lisse ou que $F$ est admissible (1.1.1). Alors $X(F)$ est dense dans $X(\widehat{F})$. 


\section{FibRÉS PRINCIPAUX SUR LES CORPS VALUÉS HENSÉLIENS}

Démonstration. Dans les deux cas, les énoncés 3.2.5 et 3.2.6 permettent de se ramener au cas où $X$ est une variété.

Lorsque $F$ est admissible, le résultat est alors démontré dans [MB12b, corollaire 1.2.1] (comme conséquence du théorème d'approximation fort, voir 4.2.2 plus bas).

Supposons désormais $X$ lisse. On peut en outre supposer $X$ affine et purement de dimension $d \geqslant 1$. Soient $\Omega$ un ouvert non vide de $X(\widehat{F})_{\text {top }}$ et $x \in \Omega$. Remplaçant $X$ par un voisinage affine de $x$, on peut supposer qu'il existe un morphisme étale $f: X \rightarrow \mathbf{A}_{F}^{d}$. Comme $\widehat{F}$ est hensélien, la proposition 3.1.4 montre que l'application $f_{\text {top }}: X(\widehat{F})_{\text {top }} \rightarrow \widehat{F}^{d}$ est ouverte. En particulier $f_{\text {top }}(\Omega)$ est ouvert dans $\widehat{F}^{d}$, donc rencontre $F^{d}$ car $F$ est dense dans $\widehat{F}$. Autrement dit, $\Omega$ rencontre $f_{\text {top }}^{-1}\left(F^{d}\right)$. Mais comme $F$ est séparablement fermé dans $\widehat{F}$ et $f$ étale, on a $f_{\text {top }}^{-1}\left(F^{d}\right)=X(F)$, d'où $X(F) \cap \Omega \neq \emptyset$.

3.5.3 Proposition. Soit $(K, v)$ un corps valué hensélien, de complété $\widehat{K}$. Soit $G$ un $K$-schéma en groupes localement de type fini. On notera

$$
\rho^{i}: \mathrm{H}^{i}(K, G) \rightarrow \mathrm{H}^{i}(\widehat{K}, G)
$$

l'application naturelle, définie pour $i \in\{0,1\}$ en général et pour $i \in \mathbb{N}$ si $G$ est commutatif.

(1) Si $K$ est admissible, $\rho^{1}$ est injective.

(2) Si $G$ est lisse, $\rho^{1}$ est bijective.

(3) Si $G$ est commutatif, $\rho^{i}$ est bijective pour tout $i \geqslant 2$.

Démonstration. (1) L'argument de torsion habituel nous ramène à établir la trivialité du noyau de l'application $H^{1}(K, G) \rightarrow H^{1}(\widehat{K}, G)$. Soit donc $E$ un $G$-torseur tel que $E(\widehat{K}) \neq \emptyset$. Alors $E$ est automatiquement un $K$-espace algébrique séparé et localement de type fini; il admet donc un sous-espace ouvert $U$, de type fini sur $K$ et vérifiant $U(\widehat{K}) \neq \emptyset$. Le théorème d'approximation faible 3.5.2 montre que $E(K) \neq \emptyset$. On conclut que $E / K$ est le $G$-torseur trivial.

(2) Comme $G$ est lisse sur $K$, les cohomologies étale et fppf de $G$ coïncident. D'autre part tout $G$ torseur est lisse sur $K$, de sorte que l'injectivité se démontre comme en (1) en utilisant le cas lisse de 3.5.2. Pour la surjectivité, on se limite dans un premier temps au cas où $G$ est affine. On plonge alors $G$ dans un $K$-groupe linéaire $\mathrm{GL}(V)$ et on pose $X=\mathrm{GL}(V) / G$. Suivant le théorème 90 de Hilbert, $\mathrm{H}_{\text {et }}^{1}(K, \mathrm{GL}(V))=1$ et $\mathrm{H}_{\text {et }}^{1}(\widehat{K}, \mathrm{GL}(V))=1$. La suite exacte courte de cohomologie galoisienne [Ser94, I.5.4] produit donc un carré commutatif exact d'ensembles pointés

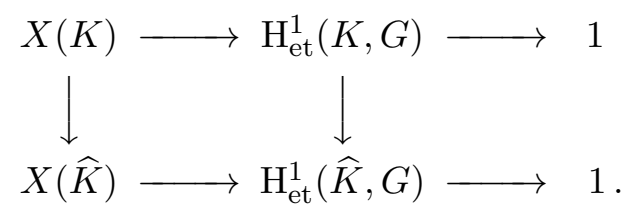

On a vu que l'application $X(\widehat{K})_{\text {top }} \rightarrow \mathrm{H}_{\text {et }}^{1}(\widehat{K}, G)$ est localement constante (lemme 3.4.1.1). Comme $X(K)$ est dense dans $X(\widehat{K})_{\text {top }}$ d'après 3.5.2, il suit que le composé $X(K) \rightarrow X(\widehat{K}) \rightarrow$ $\mathrm{H}_{\text {et }}^{1}(\widehat{K}, G)$ est surjectif. Le diagramme ci-dessus permet de conclure que l'application $\mathrm{H}^{1}(K, G) \rightarrow$ $\mathrm{H}_{\mathrm{et}}^{1}(\widehat{K}, G)$ est surjective, donc bijective.

Il reste à montrer la surjectivité de la restriction $\mathrm{H}_{\mathrm{et}}^{1}(K, G) \rightarrow \mathrm{H}_{\mathrm{et}}^{1}(\widehat{K}, G)$ pour $G / K$ lisse quelconque. Soit $\widehat{\gamma} \in \mathrm{H}_{\text {et }}^{1}(\widehat{K}, G)$, et soit $L$ une extension finie séparable de $\widehat{K}$ qui trivialise $\widehat{\gamma}$. D'après 3.5.1, il existe une extension finie séparable $K^{\prime}$ de $K$ telle que $L=\widehat{K} \otimes_{K} K^{\prime}$, qui est aussi le complété $\widehat{K^{\prime}}$ de $K^{\prime}$. 
Le quotient $X=\left(\prod_{K^{\prime} / K} G\right) / G$ est représentable par un $K$-schéma [SGA3, $\left.\mathrm{VI}_{A} .3 .2\right]$. On observe que $X$ est une $K$-forme de $G^{\left[K^{\prime}: K\right]-1}$ donc est lisse. On a le diagramme commutatif d'ensembles pointés

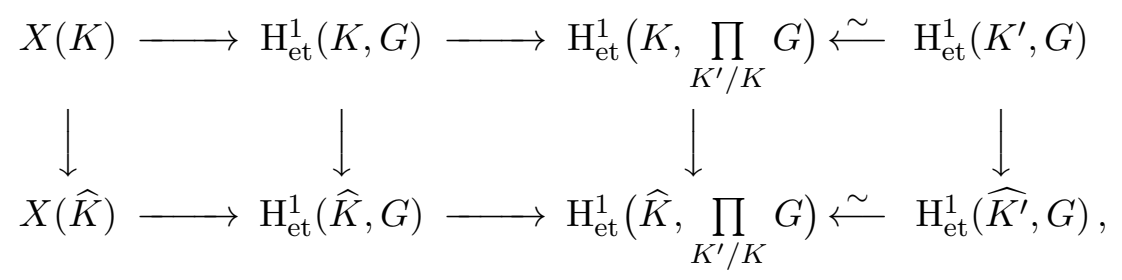

où dans chaque ligne la suite des deux premières flèches est exacte, et où les bijections de droite sont celles « de Shapiro»[SGA3, XXIV.8.4]. L'application $X(\widehat{K})_{\text {top }} \rightarrow H_{\text {et }}^{1}(\widehat{K}, G)$ est localement constante et $X(K)$ est dense dans $X(\widehat{K})_{\text {top }}$, donc $X(K)$ se surjecte sur $\mathrm{H}^{1}\left(\widehat{K^{\prime}} / \widehat{K}, G\right)$, qui contient $\widehat{\gamma}$ vu le choix de $K^{\prime}$. Le diagramme ci-dessus montre que $\widehat{\gamma}$ provient de $\mathrm{H}_{\text {et }}^{1}(K, G)$.

(3) Premier cas : $G$ est lisse. On va utiliser l'argument classique de décalage par récurrence sur $i \geqslant 2$ en notant que le cas $i=1$ a été traité. Pour tout corps $F / K$, on a $\mathrm{H}_{\mathrm{et}}^{i}(F, G) \stackrel{\sim}{\longrightarrow}$ $\mathrm{H}_{\mathrm{fppf}}^{i}(F, G)$ pour tout $i \geqslant 0$ (voir [Gro68, 11.7] ou [Mil80, III.3.9 et 3.11.(b)]). En particulier $\mathrm{H}^{i}(K, G)$ coïncide avec la cohomologie galoisienne $\mathrm{H}^{i}\left(\mathrm{Gal}\left(K_{s} / K\right), G\left(K_{s}\right)\right)$ et commute aux limites inductives filtrantes de $K$-corps. Soit donc $K^{\prime} / K$ une extension finie séparable et considérons le $K$-groupe quotient (lisse) $H=\left(\prod_{K^{\prime} / K} G\right) / G$. La suite exacte de $K$-groupes

$$
0 \rightarrow G \rightarrow \prod_{K^{\prime} / K} G \rightarrow H \rightarrow 0
$$

donne lieu au diagramme commutatif exact

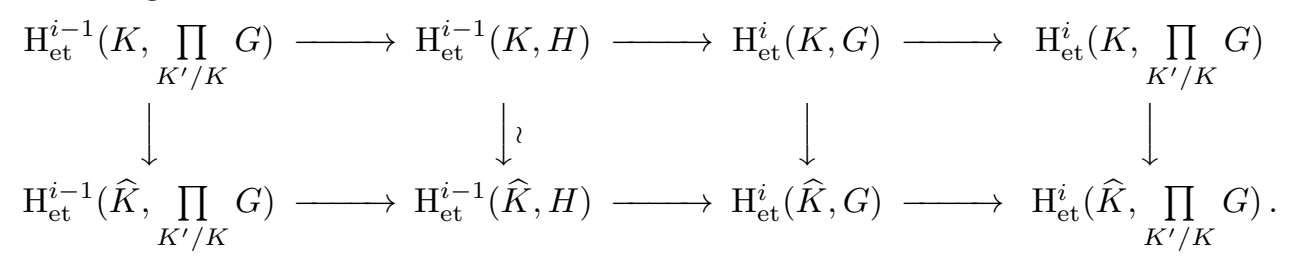

La seconde flèche verticale (en partant de la gauche) est bijective suivant l'hypothèse de récurrence. La première l'est également en tenant compte du «lemme de Shapiro » [Ser94, I.2.5]. Par suite l'application

$$
\operatorname{ker}\left(\mathrm{H}_{\mathrm{et}}^{i}(K, G) \rightarrow \mathrm{H}_{\mathrm{et}}^{i}\left(K^{\prime}, G\right)\right) \rightarrow \operatorname{ker}\left(\mathrm{H}_{\mathrm{et}}^{i}(\widehat{K}, G) \rightarrow \mathrm{H}_{\mathrm{et}}^{i}\left(\widehat{K} \otimes_{K} K^{\prime}, G\right)\right)
$$

est bijective. En passant à limite sur les sous-extensions finies $K^{\prime} / K$ de $K_{s}$, on obtient que

$$
\mathrm{H}_{\mathrm{et}}^{i}(K, G) \stackrel{\sim}{\longrightarrow} \operatorname{ker}\left(\mathrm{H}_{\mathrm{et}}^{i}(\widehat{K}, G) \rightarrow \mathrm{H}_{\mathrm{et}}^{i}\left(\widehat{K} \otimes_{K} K_{s}, G\right)\right) .
$$

Suivant 3.5.1, le corps $\widehat{K} \otimes_{K} K_{s}$ est séparablement clos, d'où la bijection souhaitée $\mathrm{H}_{\text {et }}^{i}(K, G) \stackrel{\sim}{\longrightarrow}$ $\mathrm{H}_{\mathrm{et}}^{i}(\widehat{K}, G)$.

Second cas : $G$ est fini. Soit $A$ l'algèbre affine du dual de Cartier de $G$. Alors $G$ se plonge canoniquement dans le groupe des unités $U$ de $A$, qui est un ouvert de Zariski du $K$-espace affine sous-jacent à $A$ et est donc lisse. On a donc une suite exacte $1 \rightarrow G \rightarrow U \rightarrow U / G \rightarrow 1$. Les groupes $U$ et $U / G$ étant lisses commutatifs, ils sont justiciables du cas précédent et l'on conclut par dévissage.

Cas général. En caractéristique nulle, $G$ est lisse et le premier cas suffit. Si $K$ est de caractéristique $p>0$, il existe un entier $n \geqslant 1$ tel que le quotient de $G^{\prime}=G / \operatorname{Fr}^{n} G$ par le noyau du morphisme 


\section{FibRÉS PRINCIPAUX SUR LES CORPS VALUÉS HENSÉLIENS}

de Frobenius itéré soit lisse [SGA3, $\mathrm{VII}_{A}$.8.3]. Ce noyau $N=\operatorname{Fr}^{n} G$ est fini sur $K$. En tenant compte des cas précédents, la suite exacte $1 \rightarrow N \rightarrow G \rightarrow G^{\prime} \rightarrow 1$ donne donc le résultat par dévissage.

3.5.4 Remarques. (a) L'assertion (1) est fausse si $K$ est hensélien mais non admissible. Plus généralement, soit $E \subset F$ une extension de corps non séparable, de caractéristique $p$. Alors il existe une suite $\left(a_{1}, \ldots, a_{n}\right)$ dans $E$, libre sur $E^{p}$ mais liée sur $F^{p}$, avec $n$ minimal. Considérons le $E$-groupe algébrique $G$ noyau de l'homomorphisme

$$
f:\left(x_{1}, \ldots, x_{n-1}\right) \mapsto \sum_{i=1}^{n-1} x_{i}^{p} a_{i}
$$

de $\mathbb{G}_{a}^{n-1}$ dans $\mathbb{G}_{a}$. Alors l'équation $f\left(x_{1}, \ldots, x_{n-1}\right)=a_{n}$ définit un $G$-torseur non trivial qui est trivialisé par $F$. Ainsi l'application naturelle $\mathrm{H}^{1}(E, G) \rightarrow \mathrm{H}^{1}(F, G)$ n'est pas injective.

(b) L'assertion (2) est fausse en général pour un $K$-groupe non lisse comme l'indique le contreexemple suivant. On note $A$ l'hensélisé de $\mathbb{F}_{p}[t]$ en 0 et $K$ son corps de fractions. Alors le complété de $K$ est $\mathbb{F}_{p}((t))$. La flèche $K / K^{p} \rightarrow \widehat{K} /(\widehat{K})^{p}$ n'est pas surjective car le membre de gauche est dénombrable alors que le membre de droite ne l'est pas.

(c) Lorsque $G$ est lisse ou $K$ admissible (cas (1) et (2)), le théorème d'approximation faible 3.5.2, appliqué à $G$, peut être vu comme le cas $i=0:$ l'application $\rho^{0}: G(K) \rightarrow G(\widehat{K})$ (évidemment injective) est d'image dense pour la topologie naturelle sur $G(\widehat{K})$.

\section{Corps valués admissibles; le cas d'un groupe $G$ tel que $G_{\text {red }}^{\circ}$ soit lisse}

\subsection{Corps valués admissibles : généralités}

Donnons d'abord quelques propriétés élémentaires des corps valués admissibles, définis en 1.1.1; elles ne seront pas utilisées ici, et nous en laissons au lecteur les détails des démonstrations.

4.1.1 Proposition. Soit $(K, v)$ un corps valué admissible, de complété $\widehat{K}$ et d'exposant caractéristique $p$.

(1) $K$ est algébriquement fermé dans $\widehat{K}$.

(2) L'endomorphisme $x \mapsto x^{p}$ de $K$ est fermé.

(3) Si $L$ est une extension finie de $K$ (munie de sa valuation prolongeant $v$, cf. 3.5.1), alors :

(a) $L$ est admissible;

(b) l'homomorphisme canonique $\widehat{K} \otimes_{K} L \rightarrow \widehat{L}$ est un isomorphisme;

(c) comme $K$-espace vectoriel topologique, $L$ est topologiquement libre (c'est-à-dire isomorphe à $K^{\oplus[L: K]}$ ) ; en particulier tout sous-espace vectoriel de $L$ (et notamment $K$ ) est fermé dans $L$.

Donnons seulement quelques indications sur la preuve de l'assertion (3). On sait d'après [Bou75, VI.8.2] que l'homomorphisme de la partie (3)(b) est surjectif et a pour noyau le nilradical de $\widehat{K} \otimes_{K} L$; puisque $\widehat{K} / K$ est séparable, la partie (3)(b) en résulte (et entraîne immédiatement la partie (3)(a)). Pour montrer la partie (3)(c), rappelons d'abord qu'elle est vraie si $K$ est complet : sur un corps valué complet non discret, tout espace vectoriel topologique séparé de dimension finie est libre [Bou75, VI.5.2, proposition 4]. Soit maintenant $\varphi: L \rightarrow K$ une forme $K$-linéaire, et montrons que $\varphi$ est continue (ce qui suffit pour montrer la partie (3)(c)). L'assertion (3)(b) implique que $\varphi$ se prolonge en une forme $\widehat{K}$-linéaire $\varphi_{\widehat{K}}: \widehat{L} \rightarrow \widehat{K}$; celle-ci est automatiquement continue d'après le cas complet, donc $\varphi$ l'est aussi par restriction. 


\section{Ofer Gabber, Philippe Gille et Laurent Moret-Bailly}

\subsection{Le théorème d'approximation fort; applications}

4.2.1 Notations. On désigne par $(K, v)$ un corps valué admissible, par $A$ l'anneau de $v$, par $k$ son corps résiduel et par $\widehat{K}$ le complété de $K$.

4.2.2 ThÉORÈme ( «théorème d'approximation fort »). Sous les hypothèses de 4.2.1, soit $\mathscr{Z}$ un $A$-espace algébrique de présentation finie. Pour tout idéal non nul $J$ de $A$, il existe un idéal non nul $J^{\prime} \subset J$ tel que $\mathscr{Z}(A)$ et $\mathscr{Z}\left(A / J^{\prime}\right)$ aient même image dans $\mathscr{Z}(A / J)$.

Démonstration. Lorsque $\mathscr{Z}$ est un schéma, le théorème est établi dans [Gre66] pour les valuations discrètes et dans [MB12b] pour le cas général.

Sinon, il existe d'après 3.2.5 un morphisme étale $\pi: \mathscr{Z}^{\prime} \rightarrow \mathscr{Z}$, où $\mathscr{Z}^{\prime}$ est un schéma affine, tel que (notamment) l'application induite $\pi_{k}: \mathscr{Z}^{\prime}(k) \rightarrow \mathscr{Z}(k)$ soit surjective.

Or, comme $\pi$ est étale et que $A$ (et donc tout quotient de $A$ ) est hensélien, le système projectif $\left(\mathscr{Z}^{\prime}(A / J)\right)_{J}$ (où $J$ parcourt les idéaux stricts de $A$ ) se déduit du système projectif $(\mathscr{Z}(A / J))_{J}$ par le changement de base surjectif $\pi_{k}$. L'énoncé est une conséquence formelle de cette remarque et du cas des schémas (appliqué à $\mathscr{Z}^{\prime}$ ).

4.2.2.1 Remarque. Dans l'énoncé de 4.2.2, on peut prendre $J^{\prime}$ de la forme $c J^{n}$, où $n \in \mathbb{N}_{>0}$ et $c \in A \backslash\{0\}$ sont indépendants de $J$; nous n'aurons pas à utiliser ce fait.

Le théorème 4.2.2 a la conséquence suivante, essentielle pour la preuve de 1.2.

4.2.3 Théorème. Sous les hypothèses de 4.2.1, soit $f: X \rightarrow Y$ un morphisme propre de $K$ espaces, et soit $y$ un point de $Y(K)$. On suppose que la fibre $C:=f_{\text {top }}^{-1}(y) \subset X_{\text {top }}$ est compacte (observer qu'elle est a priori séparée puisqu'elle s'identifie à $\left(X_{y}\right)_{\text {top }}$, cf. 3.2.7.1(3)).

Alors tout voisinage $U$ de $C$ dans $X_{\text {top }}$ contient un voisinage de la forme $f_{\text {top }}^{-1}(V)$, où $V$ est un voisinage de $y$ dans $Y_{\text {top }}$.

De façon équivalente, si $\Phi \subset X_{\text {top }}$ est un fermé disjoint de $C$, alors y n'est pas adhérent à $f_{\text {top }}(\Phi)$.

Démonstration. Commençons par le cas où $Y$ est une $K$-variété, que l'on peut supposer affine, la question étant locale sur $Y_{\text {top. }}$ Choisissons alors un $A$-schéma affine $\mathscr{Y}$, de présentation finie, de fibre générique $Y$, tel que $y$ se prolonge en un point $\widetilde{y} \in \mathscr{Y}(A)$. Alors $\mathscr{Y}(A)$ est un voisinage de $y$ (identifié à $\widetilde{y}$ ) dans $Y(K)$; quitte à restreindre $U$, on peut supposer que $U \subset f_{\text {top }}^{-1}(\mathscr{Y}(A))$.

4.2.3.1 Lemme. Il existe un $A$-espace $\mathscr{X}$ de présentation finie, de fibre générique $X$, et un $A$-morphisme propre $\varphi: \mathscr{X} \rightarrow \mathscr{Y}$ tel que $\varphi_{K}: \mathscr{X}_{K} \rightarrow \mathscr{Y}_{K}$ s'identifie à $f$.

Démonstration. C'est essentiellement le théorème de compactification de Nagata (voir [CLO09, Theorem 1.2.1], ou [Con07, Theorem 4.1] pour le cas des schémas), mais il faut prendre garde que le morphisme naturel $X \rightarrow \mathscr{Y}$ n'est pas en général de type fini. Il faut commencer par prolonger $X$ en un $\mathscr{Y}$-espace de présentation finie $\mathscr{X}_{1}$, de fibre générique $X$ : il suffit ensuite d'appliquer le théorème cité à $\mathscr{X}_{1} \rightarrow \mathscr{Y}$.

Lorsque $X$ est un schéma, l'existence de $\mathscr{X}_{1}$ résulte des théorèmes généraux de [EGAIV, $\S 8$ ]. Pour un espace algébrique, on écrit $X$ comme quotient d'un $Y$-schéma $X^{\prime}$ par une $Y$-relation d'équivalence étale $R \rightrightarrows X$, et l'on applique les résultats de loc. cit. au diagramme en question.

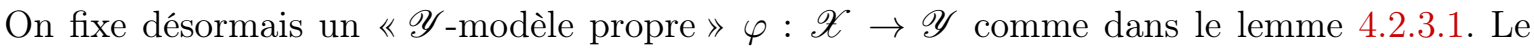
critère valuatif de propreté implique alors que $f_{\text {top }}^{-1}(\mathscr{Y}(A))=\mathscr{X}(A)$ (vus comme sous-ensembles 


\section{FibRÉS PRINCIPAUX SUR LES CORPS VALUÉS HENSÉLIENS}

de $X(K))$; nous savons en outre par 3.3.4(4) que les sous-espaces de $X_{\text {top }}$ et $Y_{\text {top }}$ ainsi définis coïncident avec les espaces $\mathscr{X}_{A \text {,top }}$ et $\mathscr{Y}_{A \text {,top }}$ définis en 3.3.1. Nous pouvons donc, dans l'énoncé, remplacer $X_{\text {top }}$ et $Y_{\text {top }}$ par $\mathscr{X}_{A \text {,top }}$ et $\mathscr{Y}_{A \text {,top }}$; en particulier, une base d'ouverts de $\mathscr{X}_{A \text {,top }}$ est fournie par les boules $B_{\mathscr{X}}(\xi, J)(\xi \in \mathscr{X}(A), J$ idéal non nul de $A)$ définies en 3.3.1.

Nous noterons $\mathscr{X}_{\widetilde{y}}$ le produit fibré $\mathscr{X} \times \mathscr{Y}, \widetilde{y} \operatorname{Spec}(A)$ : c'est un sous-espace fermé de $\mathscr{X}$ et un $A$-schéma propre, et $\mathscr{X}_{\widetilde{y}}(A)$ s'identifie à $C$. La compacité de $C$ implique donc que, quitte à restreindre $U$, on peut supposer que celui-ci est, pour $J$ convenable, de la forme

$$
\begin{aligned}
U & =\bigcup_{\xi \in C} B_{\mathscr{X}}(\xi, J) \\
& =\left\{\xi^{\prime} \in \mathscr{X}(A) \mid \exists \xi \in \mathscr{X}_{\widetilde{y}}(A), \xi \bmod J=\xi^{\prime} \bmod J\right\} .
\end{aligned}
$$

Le théorème d'approximation 4.2.2, appliqué avec $\mathscr{Z}=\mathscr{X}_{\widetilde{y}}$, fournit un idéal $J^{\prime} \subset J$ de $A$ tel que $\mathscr{X}_{\widetilde{y}}\left(A / J^{\prime}\right)$ et $\mathscr{X}_{\widetilde{y}}(A)$ aient la même image dans $\mathscr{X}_{\widetilde{y}}(A / J)$.

Posons alors $V:=B_{\mathscr{Y}}\left(\widetilde{y}, J^{\prime}\right)$; soit $\xi \in \varphi^{-1}(V)$, et vérifions que $\xi \in U$. Par hypothèse, $\varphi\left(\xi \bmod J^{\prime}\right)=\widetilde{y} \bmod J^{\prime}$, de sorte que $\xi \bmod J^{\prime} \in \mathscr{X}_{\widetilde{y}}\left(A / J^{\prime}\right)$. Vu le choix de $J^{\prime}$, son image dans $\mathscr{X}_{\widetilde{y}}(A / J)$, qui est évidemment $\xi \bmod J$, se relève en un point de $\mathscr{X}_{\widetilde{y}}(A)$; autrement dit, on a $\xi \in U$, comme annoncé.

Ne supposant plus que $Y$ est un schéma, choisissons une $K$-variété affine $Y^{\prime}$ et un morphisme étale $\pi: Y^{\prime} \rightarrow Y$ tel que $y$ se relève en $y^{\prime} \in Y^{\prime}(F)$. Considérons le produit fibré $X^{\prime}:=X \times_{Y} Y^{\prime}$ et le diagramme correspondant d'espaces topologiques

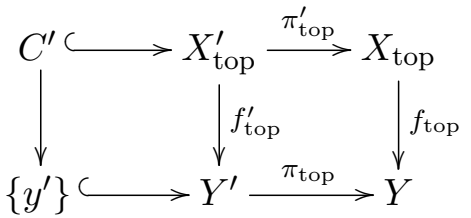

où l'on a posé $C^{\prime}=f_{\text {top }}^{\prime-1}\left(y^{\prime}\right) \subset X^{\prime}$; on sait (3.2.7.1) que $\pi_{\text {top }}^{\prime}$ induit un homéomorphisme de $C^{\prime}$ sur $C$, de sorte que $C^{\prime}$ est compact.

Posons $U^{\prime}=\pi_{\text {top }}^{\prime-1}(U)$ : c'est un voisinage de $C^{\prime}$ dans $X_{\text {top }}^{\prime}$. Il contient donc, d'après le cas déjà établi, un voisinage de la forme $f_{\text {top }}^{\prime-1}\left(W^{\prime}\right)$, où $W^{\prime} \subset Y_{\text {top }}^{\prime}$ est un ouvert contenant $y^{\prime}$. On a donc

$$
C=\pi_{\text {top }}^{\prime}\left(C^{\prime}\right) \subset \pi_{\text {top }}^{\prime}\left(f_{\text {top }}^{\prime-1}\left(W^{\prime}\right)\right) \subset \pi_{\text {top }}^{\prime}\left(U^{\prime}\right) \subset U .
$$

Bien que le diagramme ci-dessus ne soit pas nécessairement cartésien, le diagramme sous-jacent d'ensembles l'est, de sorte que

$$
\pi_{\text {top }}^{\prime}\left(f_{\text {top }}^{\prime-1}\left(W^{\prime}\right)\right)=f_{\text {top }}^{-1}\left(\pi_{\text {top }}\left(W^{\prime}\right)\right) .
$$

Mais $\pi_{\text {top }}$ est ouverte puisque $\pi$ est étale (3.2.8) donc $V:=\pi_{\text {top }}\left(W^{\prime}\right)$ est un voisinage ouvert de $y$ dans $Y_{\text {top }}$, et les relations qui précèdent montrent que $f_{\text {top }}^{-1}(V) \subset U$, ce qui achève la démonstration.

4.2.4 Corollaire. [MB12b, 1.3] Sous les hypothèses de 4.2.1, soit $f: X \rightarrow Y$ un morphisme propre de $K$-espaces. Alors l'image de $f_{\text {top }}$ est fermée dans $Y_{\text {top }}$.

Démonstration. Appliquer le théorème 4.2 .3 au fermé $\Phi=X_{\text {top }}$ et à un point $y \notin \operatorname{Im}\left(f_{\text {top }}\right)$.

4.2.5 Corollaire. Sous les hypothèses de 4.2.1, soit $f: X \rightarrow Y$ un morphisme propre de $K$-espaces.

(1) Posons $Z:=\left\{z \in Y_{\text {top }} \mid f_{\text {top }}^{-1}(z)\right.$ est compact $\}$. Alors la restriction $f_{\text {top }, Z}: f_{\text {top }}^{-1}(Z) \rightarrow Z$ de $f_{\text {top }}$ au-dessus de $Z$ est topologiquement propre. 


\section{Ofer Gabber, Philippe Gille et Laurent Moret-Bailly}

(2) Posons $Z_{1}:=\left\{z \in Y_{\text {top }} \mid\right.$ Card $\left.\left(f_{\text {top }}^{-1}(z)\right)=1\right\}$. Alors la restriction $f_{\text {top }, Z_{1}}$ de $f_{\text {top }}$ au-dessus de $Z_{1}$ est un homéomorphisme.

Démonstration. Pour l'assertion (1), il suffit de montrer que $f_{\text {top }, Z}$ est fermée (puisqu'elle est à fibres compactes, cf. [Bou71, I, $\S 10, \mathrm{n}^{\circ} 2$, théorème 1]). Or c'est une conséquence immédiate de la dernière assertion de 4.2.3. On en déduit l'assertion (2) puisque $f_{\text {top, } Z_{1}}$ est bijective par construction, et elle est propre d'après l'assertion (1) car $Z_{1} \subset Z$.

4.2.6 Corollaire. Sous les hypothèses de 4.2.1, soit $f: X \rightarrow Y$ un morphisme fini de $K$ espaces. Alors $f_{\text {top }}$ est fermée (et donc propre).

4.2.7 Remarque. Le corollaire 4.2.6 peut s'obtenir de manière plus directe et élémentaire : pour le cas d'une valuation de rang 1, voir par exemple [MB12a, proposition 2.2.1].

\subsection{Groupes $G$ tels que $G_{\text {red }}^{\circ}$ soit lisse}

On désigne par $F$ un corps topologiquement hensélien et vérifiant la propriété suivante : pour tout morphisme fini $f: X \rightarrow Y$ de $F$-variétés, l'application $f_{\text {top }}: X_{\text {top }} \rightarrow Y_{\text {top }}$ est fermée.

D'après 4.2.6, ces conditions sont vérifiées notamment si $F$ est un corps valué admissible.

4.3.1 Théorème. Soit $G$ un $F$-groupe algébrique tel que le $F$-schéma $G_{\text {red }}^{\circ}$ soit lisse (c'est donc automatiquement un sous- $F$-groupe de $\left.G^{\circ}\right)$. Soient $Y$ un $F$-espace et $f: X \rightarrow Y$ un $G$-torseur.

Alors $f_{\text {top }}: X_{\text {top }} \rightarrow Y_{\text {top }}$ est ouverte sur son image; en outre celle-ci est fermée dans $Y_{\text {top }}$, et elle est ouverte si $F$ est parfait.

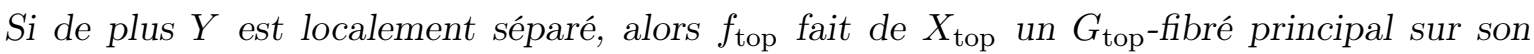
image.

4.3.1.1 Remarque (cf. remarque 3.4.2.1). Dans le cas d'un corps valué admissible, cet énoncé est, tout comme 3.4.2, conséquence du théorème principal 1.2, qui sera établi sans en faire usage.

Démonstration de 4.3.1. La question étant locale sur $Y_{\text {top }}$, nous pouvons supposer que $Y$ est un schéma.

On a $G_{\text {red }}^{\circ} \subset G^{\natural}$, de sorte que $G / G^{\natural}$ est fini sur $F$.

Posons $Z:=X / G^{\natural}$, de sorte que $f$ se décompose en $X \stackrel{h}{\rightarrow} Z \stackrel{g}{\rightarrow} Y$. Comme $G^{\natural}$ est lisse et $F$ topologiquement hensélien, la proposition 3.4.1 montre que $h_{\text {top }}$ est ouverte et que son image $Z_{\text {top }}^{\prime}$ est (ouverte et) fermée dans $Z_{\text {top }}$.

D'autre part $g$ est un morphisme fini (et en particulier $Z$ est un schéma), donc $g_{\text {top }}$ est fermée ; en outre, le corollaire 2.4.2 nous dit qu'elle est injective. C'est donc un homéomorphisme sur un

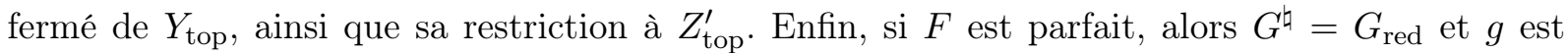
radiciel, donc ( $F$ étant parfait) $g_{\text {top }}$ est bijective et est donc un homéomorphisme : l'image de $f_{\text {top }}$ s'identifie via $g_{\text {top }}$ à celle, ouverte, de $h_{\text {top }}$.

Enfin, si $Y$ est localement séparé, alors $Z$ l'est aussi puisque $g$ est un morphisme séparé, donc $X_{\text {top }}$ est un $G_{\text {top }}$-fibré principal au-dessus de $Z_{\text {top }}^{\prime}$ (3.4.1 à nouveau).

4.3.2 Corollaire. Soient $G$ un $F$-groupe algébrique et $f: X \rightarrow Y$ un $G$-torseur, où $X$ et $Y$ sont des $F$-espaces. On suppose que $G^{\circ}$ est de type multiplicatif. Alors $f_{\text {top }}: X_{\text {top }} \rightarrow Y_{\text {top }}$ est ouverte sur son image, qui est fermée dans $Y_{\text {top }}$; si $Y$ est localement séparé, c'est même un $G_{\text {top-fibré }}$ principal sur cette image.

Démonstration. Cela résulte de 4.3.1 et du fait que $G_{\text {red }}^{\circ}$ est lisse. 


\section{FibRÉS PRINCIPAUX SUR LES CORPS VALUÉS HENSÉLIENS}

\section{Un théorème de compactification}

On désigne par $k$ un corps quelconque, et par $k_{s}$ une clôture séparable de $k$; les résultats de cette section n'ont d'intérêt que si $k$ n'est pas parfait.

5.1 Définition. Soit $X$ une $k$-variété munie d'une action d'un $k$-schéma en groupes $G$. Nous appellerons compactification $G$-équivariante de $X$ une immersion ouverte $G$-équivariante $j: X \hookrightarrow$ $X^{c}$ où $X^{c}$ est une $k$-variété propre munie d'une action de $G$.

Dans cette situation, les points et sous-schémas de $X^{c} \backslash X$ seront dits «à l'infini ».

On observera que, dans cette définition, l'immersion $j$ n'est pas supposée schématiquement dense. Nous avons fait ce choix pour la commodité de la rédaction; pour les applications où la densité est requise, il suffit de faire appel au lemme suivant.

5.1.1 Lemme. Soit $Z$ une $k$-variété munie d'une action d'un $k$-schéma en groupes $G$, et soit $Y \subset Z$ un sous-schéma stable par $G$. Alors l'adhérence schématique $\bar{Y}$ de $Y$ dans $Z$ est stable par $G$.

Démonstration. Considérons le diagramme commutatif

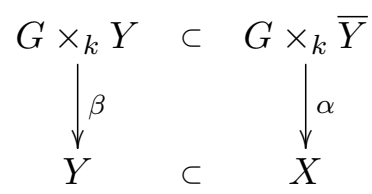

où les flèches $\alpha$ et $\beta$ sont déduites de l'action de $G$. Il s'agit de voir que $\alpha$ se factorise par $\bar{Y}$. Or $\alpha^{-1}(\bar{Y})$ est un sous-schéma fermé de $G \times_{k} \bar{Y}$ qui contient $G \times_{k} Y$, et $G \times_{k} Y$ est schématiquement dense dans $G \times_{k} \bar{Y}$ puisque l'immersion $Y \hookrightarrow \bar{Y}$ est schématiquement dense et que $G$ est plat sur $\operatorname{Spec}(k)$. Donc $\alpha^{-1}(\bar{Y})=G \times_{k} \bar{Y}$, d'où la conclusion.

Le premier auteur a annoncé des résultats de compactification généraux pour les groupes algébriques [Gab12] qui généralisent un résultat de Borel-Tits pour les groupes algébriques affines sur un corps parfait [BoT65, théorème 8.2] ainsi que le cas d'un $k$-groupe commutatif [BLR90, $\S 10.2$, théorème 7]. Nous nous intéressons ici à la variante suivante.

5.2 ThÉORÈme. Soient $G$ un k-groupe algébrique et $H=G^{\natural}$ le plus grand sous-groupe lisse de $G$ (cf. 2.4). Soit $J$ un k-groupe algébrique lisse agissant sur $G$ par automorphismes de groupe. Alors $X:=G / H$ admet une compactification $G \rtimes J$-équivariante $X^{c}$ munie d'un fibré en droites ample $G \rtimes J$-linéarisé et satisfaisant $X(E)=X^{c}(E)$ pour toute extension séparable $E$ de $k$ (ce qui équivaut à dire que $X^{c}\left(k_{s}\right)=\left\{x_{0, k_{s}}\right\}$, où $x_{0}$ désigne la classe neutre de $\left.X(k)\right)$.

Si de plus $G$ satisfait la condition $(*)$ de 2.4.3, on peut imposer en outre la condition que pour toute extension séparable $K$ de $k, X_{K}^{c}$ n'admette aucune $K$-orbite à l'infini pour l'action de $G_{K}$.

Suivant le $\S 2.4$, l'action du $k$-groupe lisse $J$ sur $G$ normalise $H$ si bien que l'on a une action de $G \rtimes_{k} J$ sur $G / H$. Si $G$ est affine et $J=1$, alors l'énoncé ci-dessus est un cas particulier de [Gab12, Theorem A]. Il se trouve que ce cas particulier est bien plus simple que le cas général, et nous en donnons une démonstration ci-dessous. 


\section{Ofer Gabber, Philippe Gille et Laurent Moret-Bailly}

\subsection{Démonstration du théorème 5.2 : dévissage}

5.3.1 Définition. Soient $k$ un corps, $k_{s}$ une clôture séparable de $k, G$ un $k$-groupe algébrique, $H$ un sous-groupe algébrique de $G$. Soit $J$ un $k$-groupe algébrique qui agit sur $G$ par automorphismes de groupes et qui normalise $H$. On note $X$ le $G$-espace homogène $G / H$.

Une bonne $G \rtimes_{k} J$-compactification de $X$ est la donnée :

- d'une $k$-variété projective $X^{c}$, munie d'une action à gauche de $G \rtimes_{k} J$,

- d'un fibré en droites ample $G \rtimes_{k} J$-linéarisé $L$ sur $X^{c}$,

— d'une immersion ouverte $G \rtimes_{k} J$-équivariante $j: X \hookrightarrow X^{c}$,

tels que le groupe $G\left(k_{s}\right)$ opère transitivement sur $X^{c}\left(k_{s}\right)$ (ce qui implique notamment que $X^{c}\left(k_{s}\right)=$ $\left.j\left(X\left(k_{s}\right)\right)\right)$.

Si de plus $X^{c}$ n'admet aucune $G$-orbite à l'infini définie sur une extension séparable de $k$, on dit que $X^{c}$ est une très bonne compactification.

5.3.2 Remarques. (1) Dans l'énoncé du théorème 5.2, la condition sur $X^{c}\left(k_{s}\right)$ équivaut trivialement à dire que l'action de $G\left(k_{s}\right)$ sur $X^{c}\left(k_{s}\right)$ est transitive. On peut donc reformuler 5.2 en disant que $X$ admet une bonne compactification, et une très bonne compactification si $G$ vérifie $(*)$.

(2) Pour démontrer 5.2, nous allons donc procéder comme suit. Si le résultat est en défaut pour $(G, H)$ et $J$, il existe un plus petit sous-groupe $G^{\prime}$ (normalisé par $J$ ) de $G$ contenant $H$ tel qu'il soit encore en défaut pour $\left(G^{\prime}, H\right)$. Remplaçant $G$ par $G^{\prime}$, nous pouvons donc supposer que pour tout sous-groupe $H^{\prime}$ de $G$ normalisé par $J$ tel que $H \subset H^{\prime} \varsubsetneqq G$, le $H^{\prime}$-espace homogène $H^{\prime} / H$ admet une bonne $H^{\prime} \rtimes J$-compactification. La construction fondamentale de la démonstration consiste alors à exhiber un tel sous-groupe $H^{\prime}$ tel que $G / H^{\prime}$ ait une bonne $G \rtimes J$-compactification; on conclut ensuite grâce à l'énoncé de dévissage 5.3 .3 cidessous, inspiré des constructions de [BLR90, § 10.2].

5.3.3 Proposition. Soient $k$ un corps, $G$ un $k$-groupe algébrique, $H \subset H^{\prime}$ des sous-groupes algébriques de $G$. Soit $J$ un k-groupe algébrique agissant sur $G$ par automorphismes de groupe en laissant stables $H$ et $H^{\prime}$.

On suppose que $G / H^{\prime}$ admet une bonne (resp. très bonne) $G \rtimes J$-compactification et que $H^{\prime} / H$ admet une bonne (resp. très bonne) $H^{\prime} \rtimes_{k} J$-compactification. Alors $G / H$ admet une bonne (resp. très bonne) $G \rtimes_{k} J$-compactification.

Démonstration. Posons $X=G / H^{\prime}, Y=G / H$, et considérons les morphismes canoniques (et $G \rtimes_{k} J$-équivariants) $G \rightarrow Y \stackrel{q}{\rightarrow} X$. Le composé $G \rightarrow X$ fait de $G$ un $H^{\prime}$-torseur à droite au-dessus de $X$.

Posons $Z=H^{\prime} / H$ (qui s'identifie à $q^{-1}\left(x_{0}\right)$ où $x_{0} \in X(k)$ est la classe neutre). Il existe par hypothèse une bonne $H^{\prime} \rtimes_{k} J$-compactification $Z \hookrightarrow Z^{c}$ de $Z$, munie notamment d'un fibré en droites ample $H^{\prime} \rtimes_{k} J$-linéarisé $N$.

On considère alors le produit contracté $Y^{c}:=G \wedge{ }_{\wedge}^{H^{\prime}} Z^{c} \rightarrow X$. D’après le $\S 2.3, Y^{c}$ est représentable par un $k$-schéma projectif sur $X$; de plus, $M:=G \stackrel{H^{\prime}}{\wedge} N$ est un fibré en droites $G \rtimes_{k} J$-linéarisé sur $Y^{c}$, ample relativement à $X$.

Par hypothèse, $X$ admet une bonne $G \rtimes_{k} J$-compactification $X^{c}$; nous avons donc un dia- 


\section{FibRÉS PRINCIPAUX SUR LES CORPS VALUÉS HENSÉLIENS}

gramme commutatif de $k$-variétés munies d'actions à gauche de $G \rtimes_{k} J$ :

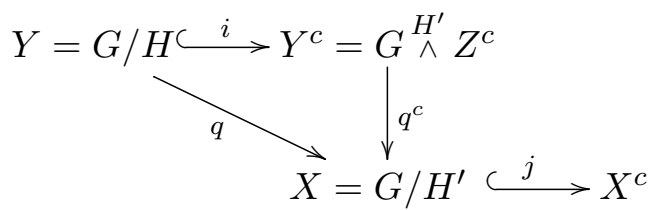

où tous les morphismes sont $G \rtimes_{k} J$-équivariants, $i$ et $j$ étant des immersions ouvertes; de plus $Y^{c}$ (resp. $X^{c}$ ) est projectif sur $X$ (resp. sur $k$ ) et est muni d'un faisceau inversible $G \rtimes J$-linéarisé $M$ (resp. $L)$, ample relativement à $X$ (resp. $k$ ). On a alors le résultat de prolongement suivant.

5.3.3.1 Lemme. Considérons un diagramme $P \stackrel{\pi}{\rightarrow} U \stackrel{j}{\rightarrow} V$ de $k$-variétés munies d'actions d'un $k$-groupe algébrique $\Gamma$, les morphismes étant $\Gamma$-équivariants. On suppose que :

- $j$ est une immersion ouverte;

- $\pi$ est projectif et $P$ admet un faisceau inversible $\Gamma$-linéarisé $\mathscr{L}$, ample relativement à $\pi$.

Alors il existe un diagramme cartésien $\Gamma$-équivariant

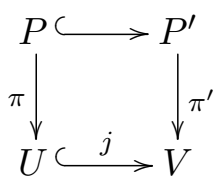

où $\pi^{\prime}$ est projectif et où $P^{\prime}$ admet un faisceau inversible $\Gamma$-linéarisé, ample relativement à $\pi^{\prime}$ et prolongeant une puissance de $\mathscr{L}$.

Démonstration. Quitte à remplacer $\mathscr{L}$ par une puissance convenable, on peut le supposer très ample relativement à $\pi$, de sorte que l'on a une $U$-immersion fermée canonique $P \hookrightarrow \mathbb{P}(\mathscr{E})$ où $\mathscr{E}=\pi_{*} \mathscr{L}$; de plus, comme $\mathscr{L}$ est $\Gamma$-linéarisé, l'action de $\Gamma$ sur $P$ est induite par une $\Gamma$ linéarisation de $\mathscr{E}$ sur $U$. D'après un lemme de prolongement de Thomason [Tho83, 2.2], il existe un $\mathscr{O}_{V}$-module cohérent $\Gamma$-linéarisé $\mathscr{E}^{\prime}$ prolongeant $\mathscr{E}$. Il suffit de prendre pour $P^{\prime}$ l'adhérence schématique de $P$ dans $\mathbb{P}\left(\mathscr{E}^{\prime}\right)$, qui est stable sous $\Gamma$ d'après 5.1.1.

Si l'on applique le lemme 5.3.3.1 au diagramme $Y^{c} \rightarrow X \rightarrow X^{c}$, avec les actions de $\Gamma=G \rtimes_{k} J$, on voit que le diagramme (1) se complète en

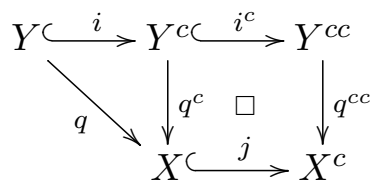

dans lequel le carré est cartésien, $q^{c c}$ est projectif et $Y^{c c}$ est muni d'un faisceau $q^{c c}$-ample $G \rtimes_{k} J$ linéarisé $M^{c}$, prolongeant une puissance de $M$. Dans ces conditions, pour $m \in \mathbb{N}$ assez grand, le faisceau inversible (évidemment $G \rtimes_{k} J$-linéarisé) $M^{c} \otimes\left(q^{c c}\right)^{*} L^{\otimes m}$ est ample sur $Y^{c c}$ [EGAII, (4.6.13)(ii)].

Il reste à montrer que $G\left(k_{s}\right)$ opère transitivement $\operatorname{sur} Y^{c c}\left(k_{s}\right)$. Soit donc $y \in Y^{c c}\left(k_{s}\right):$ montrons que $y \in G\left(k_{s}\right) . y_{0, k_{s}}$ où $y_{0}$ est la classe neutre de $Y$. L'image de $y$ dans $X^{c}$ appartient à $X^{c}\left(k_{s}\right)$ donc à l'orbite sous $G\left(k_{s}\right)$ de l'origine $x_{0, k_{s}}$ de $X\left(k_{s}\right)$, puisque $X^{c}$ est une bonne compactification. On peut donc, en faisant opérer $G\left(k_{s}\right)$, supposer que $q^{c c}(y)=x_{0, k_{s}}$. Or $\left(q^{c}\right)^{-1}\left(x_{0}\right)$ s'identifie à $Z^{c}$, et l'hypothèse de bonne compactification pour $Z$ entraîne que $H^{\prime}\left(k_{s}\right)$ opère transitivement sur $Z^{c}\left(k_{s}\right)$, de sorte que $y$ est bien dans l'orbite de $y_{0, k_{s}}$. 


\section{Ofer Gabber, Philippe Gille et Laurent Moret-Bailly}

On suppose maintenant que $X^{c}$ (resp. $Z^{c}$ ) est une très bonne compactification de $G / H^{\prime}$ (resp. $\left.H^{\prime} / H\right)$. Soit $E / k$ une extension séparable et $W \subset Y^{c c} \times_{k} E$ un sous-espace localement fermé qui soit une $G$-orbite. On veut montrer que $W \subset Y \times_{k} E$. Sans perte de généralité, il est loisible de supposer que $E=k$. La projection de $W$ par $q^{c c}$ est une $G$-orbite de $X^{c}$, donc est un sous-espace de $X$ selon notre hypothèse. Par suite $W$ est un sous-espace de $Y^{c}$. Par homogénéité, l'application $W \rightarrow X$ est surjective. On note $x_{0}$ le point privilégié de $X=G / H$. Alors la fibre $W_{x_{0}}$ est un sous-espace localement fermé de $Z^{c}$, qui est une $H^{\prime}$-orbite. Notre hypothèse implique que $W_{x_{0}} \subset Z \subset Y$. Par homogénéité, on conclut que $W \subset Y$.

\subsection{Démonstration du théorème 5.2 : construction et fin}

Posons $G_{\mathrm{af}}=\operatorname{Spec} \Gamma\left(G, \mathscr{O}_{G}\right)$ : alors d'après [SGA3, $\mathrm{VI}_{\mathrm{B}}$, théorème 12.2], le morphisme naturel $G \rightarrow G_{\text {af }}$ induit un isomorphisme $G / N \cong G_{\text {af }}$, où $N \subset G$ est distingué et lisse, et en particulier contenu dans $H$. On peut donc remplacer $G$ par $G_{\text {af }}$ et supposer que $G$ est affine (en gardant bien sûr l'action de $J$ ).

On note $k^{\prime}$ le corps de définition du $\bar{k}$-groupe lisse $\left(G \times_{k} \bar{k}\right)_{\text {red }}$; c'est une extension finie radicielle de $k$ dont on note $d$ le degré. On dispose d'une immersion fermée $G \rtimes_{k} J$-équivariante $j: G \rightarrow \prod_{k^{\prime} / k} G_{k^{\prime}}[$ CGP10, A.5.7]; on identifiera $G$ et $j(G)$. On pose

$$
\widetilde{H}=G \cap \prod_{k^{\prime} / k} G_{k^{\prime}, \text { red }} \subset \prod_{k^{\prime} / k} G_{k^{\prime}}:
$$

ce $k$-groupe contient $H$ et satisfait $H\left(k_{s}\right)=\widetilde{H}\left(k_{s}\right)$. On observe que l'action de $J$ respecte $\widetilde{H}$. En effet, le morphisme d'action $J_{k^{\prime}} \times_{k^{\prime}} G_{k^{\prime}} \rightarrow G_{k^{\prime}}$ donne lieu à un $k$-morphisme

$$
\left(J_{k^{\prime}} \times_{k^{\prime}} G_{k^{\prime}, \text { red }}\right)_{\text {red }} \stackrel{\sim}{\longrightarrow}\left(J_{k^{\prime}} \times_{k^{\prime}} G_{k^{\prime}}\right)_{\text {red }} \rightarrow G_{k^{\prime}, \text { red }} \cdot
$$

Comme $J_{k^{\prime}}$ est géométriquement réduit, $J_{k^{\prime}} \times_{k^{\prime}} G_{k^{\prime} \text {,red }}$ est réduit [GW10, 5.49.ii], d'où un morphisme $J_{k^{\prime}} \times_{k^{\prime}} G_{k^{\prime}, \text { red }} \rightarrow G_{k^{\prime}, \text { red }}$. Ainsi $J_{k^{\prime}}$ normalise $G_{k^{\prime}, \text { red }}$ et $J$ normalise $\prod_{k^{\prime} / k} G_{k^{\prime}, \text { red }}$ et $\widetilde{H}$.

5.4.1 Lemme. $\widetilde{H}=G$ si et seulement si $G$ est lisse.

Démonstration. Si $G$ est lisse, alors $k=k^{\prime}$ et

$$
\widetilde{H}=G=\prod_{k^{\prime} / k} G_{k^{\prime}}=\prod_{k^{\prime} / k} G_{k^{\prime}, \text { red }} .
$$

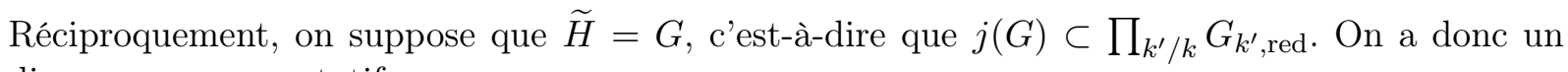
diagramme commutatif

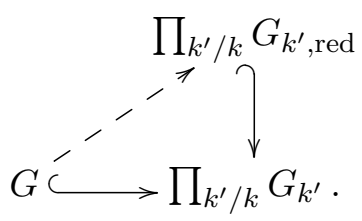

Effectuant le changement de base $k^{\prime} / k$, on obtient un diagramme commutatif

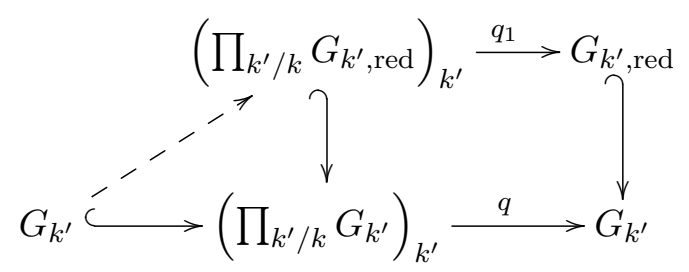




\section{FibRÉS PRINCIPAUX SUR LES CORPS VALUÉS HENSÉLIENS}

où $q$ et $q_{1}$ sont les morphismes d'adjonction [CGP10, A.5.7]. Or, la composée des flèches inférieures est l'identité de $G_{k^{\prime}}$ : on conclut que $\operatorname{Id}_{G_{k^{\prime}}}$ se factorise par l'inclusion de $G_{k^{\prime} \text {,red }}$ dans $G_{k^{\prime}}$, ce qui signifie que $G_{k^{\prime}, \text { red }}=G_{k^{\prime}}$, donc que $G$ est lisse.

Le $k^{\prime}$-schéma $Q^{\prime}:=G_{k^{\prime}} / G_{k^{\prime} \text {,red }}$ est le spectre d'une $k^{\prime}$-algèbre locale artinienne $A^{\prime}$ de corps résiduel $k^{\prime}$; on note $s$ : Spec $\left(k^{\prime}\right) \hookrightarrow Q^{\prime}$ son point fermé. Nous allons appliquer à cette situation les constructions du $\S 2.5$, dont nous reprenons les notations : ainsi, $V$ désigne le $k$-schéma sousjacent à $Q^{\prime}, W=\prod_{k^{\prime} / k} Q^{\prime}$ la restriction de Weil de $Q^{\prime}$ sur $k$, et $V^{[d]}=\operatorname{Hilb}_{V / k}^{d}$ le schéma de Hilbert des sous-schémas finis de longueur $d$ de $V$. Noter qu'ici $V$ est fini, de sorte que $V^{[d]}$ est projectif sur $k$ et admet un fibré en droites ample $G \rtimes_{k} J$-linéarisé (lemme 2.5.5), et que $W$ est affine comme restriction de Weil d'un $k^{\prime}$-schéma affine.

On a de plus une action naturelle de $G \rtimes_{k} J$ sur $V$ compatible au morphisme $\sigma: V \rightarrow \operatorname{Spec}\left(k^{\prime}\right)$ définissant le $k^{\prime}$-schéma $Q^{\prime}$.

Selon le $\S 2.5$, on dispose d'un $k$-morphisme qui est une immersion ouverte $G \rtimes_{k} J$-équivariante

$$
u: W \rightarrow V^{[d]} .
$$

En particulier, le point $s \in W(k)=Q^{\prime}\left(k^{\prime}\right)$ a pour image dans $V^{[d]}(k)$ le sous-schéma fermé réduit $\{s\}$ de $V$, qui est bien de degré $d$ sur $k$. Par abus, on notera encore $s$ le point correspondant de $W\left(k_{s}\right)$.

Observons que $V^{[d]}\left(k_{s}\right)=W\left(k_{s}\right)=\{s\}$ : ceci résulte du fait que $V \otimes_{k} k_{s}$ est local de corps résiduel $k^{\prime} \otimes_{k} k_{s}$, extension de degré $d$ de $k_{s}$.

Le groupe $G$ agit sur le couple $\left(V^{[d]}, W\right)$, et le stabilisateur de l'unique $k$-point $s$ de $V^{[d]}(k)$ est $\widetilde{H}$. Ceci produit un morphisme d'orbite $i: G / \widetilde{H} \rightarrow W$ qui est une immersion (2.1.2). Utilisons la même notation $X$ pour le quotient $G / \widetilde{H}$ et pour son image par le composé $i: G / \widetilde{H} \rightarrow$ $W \rightarrow V^{[d]}$, et notons $X^{c}$ l'adhérence schématique de $X$ dans $V^{[d]}$. Alors $X^{c}$ est une compactification $G \rtimes_{j} J$-équivariante de $X$, et c'est même une bonne $G \rtimes_{k} J$-compactification puisque $X^{c}\left(k_{s}\right)=X\left(k_{s}\right)=\{s\}$. Enfin le lemme 5.4.1 nous dit que $\widetilde{H} \varsubsetneqq G$, sauf dans le cas trivial où $G$ est lisse. Comme expliqué dans la remarque 5.3.2(2), ceci suffit à conclure compte tenu de la proposition 5.3.3.

Nous allons raffiner l'argument dans le cas où le groupe $G$ satisfait la condition $(*)$ afin de montrer que la compactification construite est très bonne. Tout d'abord, le $k$-groupe $\widetilde{H}$ satisfait lui aussi l'hypothèse (*) puisqu'il contient $G^{\natural}$ (lemme 2.4.5 (2)) et la récurrence fonctionne bien en appliquant le raffinement de la proposition 5.3.3. L'unique chose à vérifier est que $X^{c}$ n'a aucune $G$-orbite à l'infini définie sur une extension séparable de $k$. Soient donc $E$ une extension séparable de $k$ et $I \subset\left(X^{c}\right)_{E}$ une $G_{E}$-orbite. Alors $k^{\prime} \otimes_{k} E$ est un corps, donc le théorème 2.5.4 indique que $I \subset W_{E}$. Pour conclure, il suffit donc de montrer que $X=X^{c} \cap W$, c'est-à-dire que $X$ est fermé dans $W$. Comme $W$ est affine, cette assertion résulte (compte tenu de l'hypothèse $(*)$ ) du lemme 2.4.7, ce qui achève la démonstration.

\section{Démonstration du théorème 1.2}

On suppose désormais que $K$ est un corps valué admissible, et l'on se donne $G$ et $f: X \rightarrow Y$ comme dans 1.2, dont nous allons achever la preuve. Les cas particuliers où $G$ est lisse (3.4.1) et où $K$ est parfait (4.3.1) ont déjà été traités. Il reste à montrer les assertions (1)(a) et (1)(c) $\left(I \subset Y_{\text {top }}\right.$ est localement fermé, et est fermé sous la condition $\left.(*)\right),(2)\left(f_{\text {top }}\right.$ est ouverte sur $\left.I\right)$, et (3) ( $X_{\text {top }} \rightarrow I$ est un $G_{\text {top }}$-fibré principal si $Y$ est localement séparé). On fixe une clôture 


\section{Ofer Gabber, Philippe Gille et Laurent Moret-Bailly}

séparable de $K$, notée $K_{s}$.

Notons $H=G^{\natural}$ le plus grand sous-groupe lisse de $G$ (2.4). Suivant le théorème 5.2, $G / H$ admet une compactification $G$-équivariante $(G / H)^{c}$, ayant l'origine comme unique point séparable. Sous la condition $\left(^{*}\right)$, nous supposerons en outre que $(G / H)^{c}$ est une très bonne compactification.

Considérons le produit contracté $Z^{c}:=X_{\wedge}^{G}(G / H)^{c}$ : c'est une compactification relative à $Y$ (c'est-à-dire propre sur $Y$ ) de $Z:=X_{\wedge}^{G}(G / H)$; ce dernier n'est autre que le quotient $X / H$. Posons $Z^{\infty}:=Z^{c} \backslash Z$ (avec sa structure réduite, par exemple). On a un diagramme commutatif

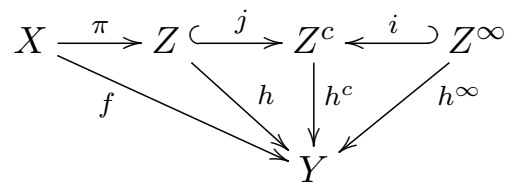

dans lequel $\pi$ est un torseur sous le groupe lisse $H, j$ est une immersion ouverte, $i$ est l'immersion fermée complémentaire, $h^{c}$ et $h^{\infty}$ sont propres.

6.1 Lemme. L'application $h_{\text {top }}: Z(K) \rightarrow Y(K)$ est injective, et les images de $h_{\text {top }}$ et de $h_{\text {top }}^{\infty}$ sont disjointes. Si de plus $(G / H)^{c}$ est une très bonne compactification (et notamment si $G$ vérifie $\left(^{*}\right)$, d'après nos conventions), alors on a $Z^{c}(K)=Z(K)$, de sorte que $\operatorname{Im}\left(h_{\mathrm{top}}\right)=\operatorname{Im}\left(h_{\mathrm{top}}^{c}\right)$.

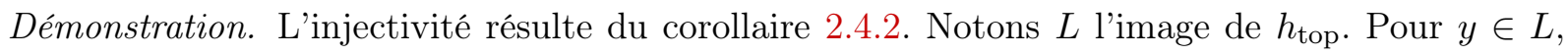
considérons les fibres $Z_{y} \subset Z_{y}^{c}$ de $h$ et $h^{c}$ en $y$ de sorte que $Z_{y}(K)$ a un seul élément $z$, qui est même le seul point séparable de $Z_{y}$. Puisque $\pi$ est lisse et surjectif, $\pi^{-1}(z)\left(K_{s}\right) \neq \emptyset$, et donc le $G$-torseur $X_{y}$ devient trivial sur $K_{s}$, de sorte que $\left(Z_{y}^{c}\right)_{K_{s}}$ est isomorphe à $(G / H)_{K_{s}}^{c}$ et a donc, lui aussi, un seul point séparable; autrement dit, $Z_{y}^{\infty}\left(K_{s}\right)=\emptyset$. Ceci montre que $L \cap \operatorname{Im}\left(h_{\text {top }}^{\infty}\right)=\emptyset$.

Supposons que $(G / H)^{c}$ soit une très bonne compactification. Soit $z \in Z^{c}(K)$ et montrons que $z \in Z(K)$. Posons $y=h^{c}(z) \in Y(K)$. Alors $Z_{y}^{c}$ s'identifie à $X_{y} \stackrel{G}{\wedge}(G / H)^{c}$ qui est muni d'une action à gauche du $K$-groupe algébrique $G^{\prime}=\underline{\operatorname{Aut}}_{G}\left(X_{y}\right)$ (2.3.2). Considèrons la $K$-orbite $T$ de $z$ dans $Z_{y}^{c}$ sous $G^{\prime}$ (remarque 2.1.4.(i)). Celle-ci correspond canoniquement, d'après 2.3.2, à une $K$-orbite $T_{0}$ de $(G / H)^{c}$ sous l'action de $G$. Vu notre hypothèse, on a $T_{0} \subset G / H$, et donc $T \subset Z_{y}$ et $z \in Z(K)$.

Ceci implique déjà que

$$
L=\operatorname{Im}\left(h_{\text {top }}^{c}\right) \backslash \operatorname{Im}\left(h_{\text {top }}^{\infty}\right)
$$

et, puisque $h^{c}$ et $h^{\infty}$ sont propres, les deux images au membre de droite sont fermées dans $Y_{\text {top }}$ (corollaire 4.2.4), de sorte que $L$ est localement fermé. Sous l'hypothèse $\left({ }^{*}\right)$, on a même $L=$ $\operatorname{Im}\left(h_{\mathrm{top}}^{c}\right)$ qui est fermé.

En outre, l'assertion (2) du corollaire 4.2.5 entraîne que $h_{\text {top }}$ est un homéomorphisme sur son image (c'est en effet la restriction de $h_{\text {top }}^{c}$ au-dessus de $L$, qui est contenu dans l'ensemble noté $Z_{1}$ dans loc. cit.).

En résumé, $f_{\text {top }}$ se décompose comme suit :

$$
X_{\text {top }} \stackrel{\pi_{\text {top }}}{\longrightarrow} U \hookrightarrow Z_{\text {top }} \stackrel{\sim}{\longrightarrow} L \hookrightarrow Y_{\text {top }}
$$

où l'on a posé $U=\operatorname{Im}\left(\pi_{\text {top }}\right) \subset Z_{\text {top }}$ et où :

- la première flèche $X_{\text {top }} \rightarrow U$ est surjective et ouverte, et est une $G_{\text {top }}$-fibration principale si $Y$, et donc $Z$, est localement séparé; 


\section{FibRÉS PRINCIPAUX SUR LES CORPS VALUÉS HENSÉLIENS}

- $U \hookrightarrow Z_{\text {top }}$ est un plongement ouvert et fermé ;

- $Z_{\text {top }} \stackrel{\sim}{\longrightarrow} L$ est un homéomorphisme;

- $L \hookrightarrow Y_{\text {top }}$ est un plongement localement fermé, et fermé sous l'hypothèse $\left(^{*}\right)$;

(bien entendu, les deux premières propriétés résultent de la proposition 3.4.1 puisque $\pi$ est un torseur sous le groupe lisse $H$, et que $\left.H_{\text {top }}=G_{\text {top }}\right)$. Ceci achève la démonstration du théorème.

\section{Exemples et compléments}

\subsection{Un exemple d'orbite topologique non fermée}

Soit $K$ un corps topologique (séparé et non discret), de caractéristique $p>0$ et non parfait; nous allons donner un exemple d'un $K$-groupe algébrique $G$ et d'un sous-groupe $H$ tels que l'image de $G(K)$ dans $(G / H)(K)$ ne soit pas fermée.

On notera $\sigma: K \rightarrow K$ l'endomorphisme de Frobenius, et $K_{0} \subset K$ son image (que l'on munira de sa topologie de sous-espace de $K$ ). On considère l'action du $K$-groupe $G:=\mathbb{G}_{a} \rtimes \mathbb{G}_{m}$ (produit semi-direct pour l'action standard de $\mathbb{G}_{m}$ sur $\mathbb{G}_{a}$ ) sur la droite affine $\mathbb{A}_{K}^{1}$ donnée par

$$
(x, y) . z=x^{p}+y^{p} z \quad\left(x \in \mathbb{G}_{a}, y \in \mathbb{G}_{m}, z \in \mathbb{A}^{1}\right) .
$$

Ainsi $\mathbb{A}_{K}^{1}$ est un $K$-espace homogène à gauche sous $G$ et le stabilisateur de 0 est le $K$-sous-groupe fermé $\alpha_{p} \rtimes_{K} \mathbb{G}_{m}$.

Fixons $z \in K \backslash K_{0}$ et considérons l'application d'orbite $\omega_{z}: g \mapsto g . z$ de $G(K)$ dans $K$. Elle est injective et son image est $G(K) . z=K_{0}+K_{0}^{\times} z \subset K$, qui ne contient pas 0 ; mais puisque $K^{\times}$ est dense dans $K$ ( $K$ est non discret), l'adhérence de $G(K)$. $z$ contient 0 , donc $G(K)$. z n'est pas fermée.

Le stabilisateur $G_{z}$ du point $z$ satisfait $G_{z}\left(K_{s}\right)=1$ et donc $G_{z}^{\natural}=1$. Par ailleurs, vu que $G$ agit transitivement sur $\mathbb{A}_{K}^{1}, G_{z}$ contient un $\bar{k}$-tore de rang un. Ainsi le groupe $G_{z}$ ne satisfait pas la condition (*) ce qui est cohérent avec le fait que le $G_{z}$-torseur $G \rightarrow G / G_{z}$ ne satisfait pas le théorème $1.2(1)(\mathrm{c})$.

7.1.1 Remarques. Le lecteur pourra vérifier les compléments suivants :

(1) (a) $G(K) . z$ est localement fermée dans $K$ si et seulement si $K_{0}$ et $E_{z}:=K_{0}+K_{0} z$ sont fermés dans $K$;

(b) pour que $\omega_{z}$ soit stricte (donc un homéomorphisme sur son image), il faut et il suffit que $\sigma$ soit un homéomorphisme sur $K_{0}$ (ce qui est toujours le cas si $K$ est un corps valué) et que $E_{z}$ soit topologiquement libre (comme $K_{0}$-espace vectoriel).

(2) L'orbite de 0 sous $G(K)$ est $K_{0}$; en particulier elle est localement fermée si et seulement si $K_{0}$ est localement fermé (donc fermé) dans $K$.

L'application d'orbite correspondante $g \mapsto g .0$ est stricte si et seulement si $\sigma$ est un homéomorphisme sur $K_{0}$; dans ce cas, $G(K) \rightarrow K_{0}$ a une section continue donnée par $t \mapsto\left(t^{1 / p}, 1\right)$ donc est un torseur trivial sous $K_{\text {top }}^{\times}$.

\subsection{Contre-exemples sur un corps valué hensélien non admissible}

Soit $A$ un anneau de valuation discrète hensélien, de caractéristique $p>0$, et soit $v \in \widehat{A}$ tel que $v \notin A$ et $v^{p} \in A$; des exemples d'une telle situation ont été construits par Nagata [Nag62, A1, (E2.1)] et par F. K. Schmidt (voir [Kuh, 11.40] ou [BLR90, §3.6, Example 11]). On note $K$ le corps des fractions de $A$, muni de la topologie de la valuation. 


\section{Ofer Gabber, Philippe Gille et Laurent Moret-Bailly}

7.2.1 Une orbite non localement fermée. Considérons le morphisme de Frobenius $\Phi$ : $\mathbb{A}_{K}^{1} \rightarrow \mathbb{A}_{K}^{1}$ : l'image $K_{0}$ de $\Phi_{\text {top }}$ n'est pas fermée puisque $v^{p}$ est adhérent à $K_{0}$, mais n'est pas dans $K_{0}$. Puisque c'est un sous-groupe de $K$, elle n'est pas non plus localement fermée.

Comme $\Phi$ est fini, ceci montre aussi que l'on ne peut pas supprimer l'hypothèse admissible dans 4.2.6.

7.2.2 Une application d'orbite non stricte. Reprenons maintenant l'exemple de 7.1 avec ce même corps $K$ et en prenant $z=v^{p}$. L'application d'orbite (injective) $\omega_{z}$ est

$$
\begin{aligned}
\omega_{z}: K \times K^{\times} & \longrightarrow K \\
(x, y) & \longmapsto x^{p}+y^{p} z
\end{aligned}
$$

qui n'est pas un homéomorphisme sur son image $K_{0}+K_{0}^{\times} z$ : en effet, soit $\left(t_{n}\right)_{n \in \mathbb{N}}$ une suite d'éléments de $K^{\times}$tels que $\lim _{n \rightarrow+\infty} t_{n}^{p}=z$. Alors la suite $\left(0,1 / t_{n}\right)$ ne converge pas dans $K \times K^{\times}$, mais son image par $\omega_{z}$ est la suite $\left(z / t_{n}^{p}\right)$ qui converge vers $1=\omega_{z}(0,1)$.

\subsection{Espaces non localement séparés.}

Soit $F$ un corps topologiquement hensélien non discret de caractéristique différente de 2 . Considérons le morphisme

$$
r: \quad R:=\mathbb{A}_{F}^{1} \amalg \mathbb{G}_{m, F} \rightarrow \mathbb{A}_{F}^{2}
$$

dont la restriction à $\mathbb{A}_{F}^{1}$ (resp. à $\mathbb{G}_{m, F}$ ) est le morphisme diagonal (resp. le morphisme $x \mapsto$ $(x,-x))$. Alors $R$ est une relation d'équivalence étale sur $\mathbb{A}_{F}^{1} ;$ considérons le faisceau étale quotient

$$
\pi: \quad \mathbb{A}_{F}^{1}=: L \rightarrow X=L / R
$$

qui est un $F$-espace. Le morphisme $r$ coïncide avec le monomorphisme naturel $L \times_{X} L \rightarrow L \times_{F} L$, de sorte que $r_{\text {top }}$ n'est pas un plongement topologique (son image est la réunion des deux diagonales de $F^{2}$ ). On en déduit par 3.2.7 (1) que la bijection $\left(L \times_{X} L\right)_{\text {top }} \rightarrow L_{\text {top }} \times_{X_{\text {top }}} L_{\text {top }}$ n'est pas un homéomorphisme.

Notons $l_{0} \in L(F)$ l'origine de $L$. La projection $\pi_{\text {top }}$ n'est pas un homéomorphisme local en $l_{0}$ puisqu'elle n'est pas localement injective (tout voisinage de $l_{0}$ contient un point $l \neq l_{0}$ et son opposé).

7.3.1 Description de $X_{\text {top }}$. Le morphisme $f: x \mapsto x^{2}$ de $\mathbb{A}_{F}^{1}$ dans $\mathbb{A}_{F}^{1}$ se factorise par $\pi$, donnant naissance à un diagramme commutatif

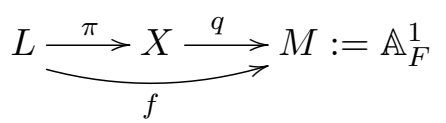

dans lequel $\pi$ est étale et surjectif et $q$ induit un isomorphisme au-dessus de $\mathbb{G}_{m, F}$. Notons $l_{0} \in L(F)$ l'origine de $L$ et $x_{0}$ et $m_{0}$ ses images dans $X$ et dans $M$, et $L^{*}\left(\operatorname{resp} . X^{*}, M^{*}\right)$ les ouverts de $L$ (resp. $X, M$ ) complémentaires de ces points.

Il est clair que $X_{\text {top }}$ est réunion de l'ouvert $X_{\text {top }}^{*}$ (qui s'identifie à $M_{\text {top }}^{*} \cong F^{\times}$par $q_{\text {top }}$ ) et de l'image $I$ de $\pi_{\text {top }}$. Comme $\pi_{\text {top }}$ est ouverte (3.2.8), $I$ est aussi un ouvert de $X_{\text {top }}$, et $q$ induit une bijection de $I$ sur l'ensemble $Q$ des carrés de $F$. Cette bijection est même un homéomorphisme (remarquer que $f_{\text {top }}$ est ouverte sur son image) de sorte que $X_{\text {top }}$ peut se décrire comme la somme topologique $X_{\text {top }}=Q \amalg_{Q^{\times}} F^{\times}$, où $Q^{\times} \subset F^{\times}$est l'ensemble des carrés non nuls. Vu les hypothèses faites sur $F, Q^{\times}$est ouvert et fermé dans $F^{\times}$, qui est donc somme disjointe de $Q^{\times}$et de $F \backslash Q$ : 


\section{FibRÉS PRINCIPAUX SUR LES CORPS VALUÉS HENSÉLIENS}

en conclusion,

$$
X_{\text {top }}=Q \amalg(F \backslash Q) \text {. }
$$

7.3.1.1 Remarque. Le morphisme $q: X \rightarrow M$ est un exemple de «bug-eyed cover $»$ au sens de [Kol92, § 4].

L'espace $X$ apparaît aussi dans [Sch02] comme exemple d'un espace algébrique admettant un $\mathbb{G}_{m}$-torseur non localement trivial pour la topologie de Zariski; ce dernier s'obtient, par le changement de groupe $\mu_{2} \hookrightarrow \mathbb{G}_{m, F}$, à partir du $\mu_{2}$-torseur que nous allons décrire plus bas (7.3.4).

7.3.2 Le cas où $F=\mathbb{R}$. Dans ce cas, la description ci-dessus montre que $X_{\text {top }}$ s'identifie (via $\left.q: X_{\text {top }} \rightarrow \mathbb{R}\right)$ à la somme disjointe des intervalles $\mathbb{R}_{<0}$ et $\mathbb{R}_{\geqslant 0}$. L'application $\pi_{\text {top }}$ s'identifie à l'application $t \mapsto t^{2}$ de $\mathbb{R}$ dans $\mathbb{R}_{\geqslant 0}$; ici, elle admet en tout point de $L_{\text {top }}$ des sections locales continues.

7.3.3 Le cas où $F=\mathbb{C}$. Alors $q_{\text {top }}: X_{\text {top }} \rightarrow \mathbb{C}$ est un homéomorphisme, et $\pi_{\text {top }}$ n'admet pas de sections locales continues au voisinage de $x_{0}$.

On remarquera que l'injection naturelle $X(\mathbb{R})_{\text {top }} \hookrightarrow X(\mathbb{C})_{\text {top }}$ n'est pas un plongement topologique.

7.3.4 Un $\mu_{2}$-torseur sur $X$. Nous allons construire un $\mu_{2}$-torseur $\alpha: \widetilde{X} \rightarrow X$ tel que l'application induite $\widetilde{X}_{\text {top }} \rightarrow \operatorname{Im}\left(\alpha_{\text {top }}\right)$ ne soit pas un $\{ \pm 1\}$-fibré principal. Considérons deux exemplaires $L_{1}$ et $L_{2}$ de $L$ (avec origines $l_{1}$ et $l_{2}$ ) et recollons-les en identifiant les ouverts $L_{1}^{*}$ et $L_{2}^{*}$ de la manière évidente. On obtient une $F$-variété non séparée $\widetilde{X}$, qui est la « droite à point dédoublé » bien connue. Il existe une unique involution $\sigma$ de $\widetilde{X}$ qui induit sur $\widetilde{X}^{*} \cong \mathbb{G}_{m}$ l'application $x \mapsto-x$, et qui échange les images de $l_{1}$ et $l_{2}$. On en déduit une action libre de $\mu_{2}$ sur $\widetilde{X}$, et il est facile de vérifier que l'application évidente $\alpha: \widetilde{X} \rightarrow X$ héritée des projections $L_{i} \cong L \stackrel{\pi}{\rightarrow} X$ $(i=1,2)$ identifie $X$ au quotient de $\widetilde{X}$ par cette action et fait de $\widetilde{X}$ un $\mu_{2}$-torseur sur $X$. L'image $I$ de $\alpha_{\text {top }}$ est celle de $\pi_{\text {top }}$, et $\widetilde{X}_{\text {top }}$ n'est pas un $\{ \pm 1\}$-fibré principal sur $I$ car $I$ est séparé et $\widetilde{X}_{\text {top }}$ ne l'est pas.

Si $F=\mathbb{R}, I$ s'identifie à $\mathbb{R}_{\geqslant 0}$ et $\widetilde{X}_{\text {top }}$ à la réunion de deux exemplaires $L_{1, \text { top }}$ et $L_{2, \text { top }}$ de $\mathbb{R}$, identifiés le long de $\mathbb{R}^{\times}$; l'application $\pi_{\text {top }}$ envoie $L_{i, \text { top }}(i=1,2)$ sur $I$ par l'application $t \mapsto t^{2}$. On voit donc que $\pi_{\text {top }}$ a quatre sections continues $s_{i, \varepsilon}(i=1,2 ; \varepsilon= \pm 1)$, où $s_{i, \varepsilon}$ envoie le point d'abscisse $u$ de $I$ sur le point d'abscisse $\varepsilon \sqrt{u}$ de $L_{i \text {,top }}$.

Si $F=\mathbb{C}, \pi_{\text {top }}$ n'a pas de section continue.

7.3.4.1 Remarque. Le $\mathbb{G}_{m}$-torseur de Schröer [Sch02], déjà mentionné en 7.3.1.1, s'obtient comme quotient de $L \times{ }_{F} \mathbb{G}_{m, F}$ par la relation d'équivalence qui identifie $(x, \lambda) \in L^{*} \times{ }_{F} \mathbb{G}_{m, F}$ à $(-x,-\lambda)$. Il donne un autre exemple où la conclusion de $1.2(3)$ est en défaut; cette fois le groupe $G$ est lisse et connexe.

7.3.5 Encore un torseur sur $X$. Pour le lecteur qui jugerait artificiels les exemples précédents, nous allons débusquer le même espace $X$ « dans la nature », comme quotient d'une honnête variété (quasi-affine) par une action de groupe. Soit $U \subset \mathbb{A}_{F}^{2}=\operatorname{Spec} F[x, y]$ l'ouvert complémentaire de l'origine. Soit $T$ le tore maximal de $\mathrm{SL}_{2, F}$ formé des matrices $\left(\begin{array}{cc}\lambda & 0 \\ 0 & \lambda^{-1}\end{array}\right)(\lambda \neq 0)$, et soit $G=T \cup \rho T$ où $\rho=\left(\begin{array}{cc}0 & -1 \\ 1 & 0\end{array}\right)$. En d'autres termes, $G$ est le sous-groupe de $\mathrm{SL}_{2, F}$ stabilisant la paire $\{h,-h\}$ où $h$ est la forme quadratique $(x, y) \mapsto x y$, et $T=G^{\circ}=\mathrm{SO}(h)$. 


\section{Ofer Gabber, Philippe Gille et Laurent Moret-Bailly}

Il est immédiat que $G$ opère librement sur $U$, mais qu'il a deux types d'orbites :

- les réunions de deux hyperboles « opposées », d'équation de la forme $(x y-a)(x y+a)=0$ $($ avec $a \neq 0)$;

- la réunion des deux axes, privée de l'origine.

Considérons d'abord le quotient de $U$ par $T$. Soient $U_{1}$ et $U_{2}$ les ouverts de $U$ complémentaires des deux axes : alors la fonction $x y$ induit un isomorphisme de $T \backslash U_{1}$ (resp. $T \backslash U_{2}$ ) sur un exemplaire $L_{1}$ (resp. $L_{2}$ ) de la droite affine, et il est facile d'en déduire un isomorphisme $T \backslash U \stackrel{\sim}{\rightarrow} \widetilde{X}$, où $\widetilde{X}$ est défini en 7.3.4. Noter que $U \rightarrow \widetilde{X}$ est localement trivial pour la topologie de Zariski et qu'en particulier l'application induite $U_{\text {top }} \rightarrow \widetilde{X}_{\text {top }}$ est un $T_{\text {top }}$-fibré principal.

La matrice $\rho$ induit sur $\tilde{X}$ l'involution $\sigma$ de 7.3 .4 : elle transforme l'hyperbole $x y=a$ en $x y=-a$, et elle échange les deux axes. Par suite le quotient $G \backslash U$ n'est autre que $X$, et $U$ est un $G$-torseur sur $X$. Ici encore, $U_{\text {top }}$ n'est pas un $G_{\text {top }}$-fibré principal sur son image, puisqu'il se factorise par $\widetilde{X}_{\text {top }} \rightarrow I$ qui n'en est pas un, et que $U_{\text {top }} \rightarrow \widetilde{X}_{\text {top }}$ est surjectif.

\subsection{Cas d'un schéma en groupes non constant}

Soient $K$ et $Y$ comme dans le théorème 1.2. Il est naturel de se demander ce que l'on peut dire de $f_{\text {top }}: X_{\text {top }} \rightarrow Y_{\text {top }}$ lorsque $f: X \rightarrow Y$ est un torseur sous un $Y$-espace en groupes $\mathfrak{G}$, non nécessairement « constant » sur $Y$ (par abus, nous appellerons constant un groupe $Y \times_{K} G$ où $G$ est un $K$-groupe).

Un cas particulier important est celui d'un groupe $\mathfrak{G}$ localement constant (au sens étale ou fppf). Même dans ce cas, la stratégie de démonstration utilisée pour 1.2 se heurte immédiatement à l'absence d'un analogue du sous-groupe $G^{\natural}$.

Dans la suite, nous supposerons en général que la base $Y$ est localement séparée; ceci assure que $\mathfrak{G}_{\text {top }} \rightarrow Y_{\text {top }}$ est un « groupe topologique relatif » opérant sur $X_{\text {top }}(3.2 .7$ (iv)).

7.4.1 Proposition. Soient $F$ un corps topologiquement hensélien, $Y$ un $F$-espace localement séparé, $\mathfrak{G}$ un $Y$-espace algébrique en groupes lisse de type fini et quasi-séparé, $f: X \rightarrow Y$ un $\mathfrak{G}$-torseur. Alors $f_{\text {top }}$ admet des sections locales en tout point de $X_{\text {top }}$; en particulier elle est ouverte et l'application induite $X_{\text {top }} \rightarrow \operatorname{Im} f_{\text {top }}$ est une $\mathfrak{G}_{\text {top }}$-fibration principale.

Démonstration. Le morphisme $f$ est lisse et $Y$ est localement séparé donc $f_{\text {top }}$ admet des sections locales $(3.2 .8(3)$ ). (L'hypothèse de quasi-séparation est là pour assurer que $\mathfrak{G}$ est un $F$-espace au sens du présent article).

7.4.2 Remarque. Dans la situation de 7.4.1, l'image de $f_{\text {top }}$ n'est pas nécessairement fermée, comme le montre l'exemple suivant. Supposant $F$ de caractéristique différente de 2, prenons pour $Y$ la droite affine Spec $F[t]$. Considérons la $\mathscr{O}_{Y}$-algèbre $\mathscr{A}:=\mathscr{O}_{Y}[Z] /\left(Z^{2}-t^{2}\right)$, qui est libre de rang 2 . Soit $\mathfrak{G}$ le groupe des unités de norme 1 de $\mathscr{A}$ : on peut le voir comme le sous-groupe de $\mathrm{GL}_{2, \mathscr{O}_{Y}}$ formé des matrices de la forme $M(x, y)=\left(\begin{array}{cc}x & t^{2} y \\ y & x\end{array}\right)$ et de déterminant 1. C'est un $Y$-groupe affine, lisse et commutatif dont la restriction à $U:=\operatorname{Spec} F\left[t, t^{-1}\right]$ est isomorphe à $\mathbb{G}_{m, U}$ (par $M(x, y) \mapsto x+t y)$ et dont la fibre à l'origine est isomorphe à $\mu_{2, F} \times \mathbb{G}_{a, F}(\operatorname{par} M(x, y) \mapsto(x, x y))$.

Soit $d \in F^{\times} \backslash F^{\times 2}$. Alors les matrices $M(x, y)$ de déterminant $d$ forment un $\mathfrak{G}$-torseur $X \rightarrow Y$. Il est trivial sur $U$ comme tout $\mathbb{G}_{m}$-torseur; une section explicite est donnée par $M((1+d) / 2,(1-d) / 2 t)$. Sa fibre à l'origine est isomorphe à $\operatorname{Spec} F(\sqrt{d}) \times \mathbb{A}_{F}^{1}$ donc n'a pas de point rationnel. Ainsi l'image de $f_{\text {top }}: X \rightarrow F$ est $F^{\times}$. 


\section{FibRÉS PRINCIPAUX SUR LES CORPS VALUÉS HENSÉLIENS}

7.4.3 Lemme. Soient $k$ un corps, $Y$ un $F$-espace, $\mathfrak{G}$ un $Y$-espace algébrique en groupes quasiséparé de type fini. On suppose que $\mathfrak{G}$ est localement isomorphe pour la topologie étale à un $Y$-groupe constant $G \times_{k} Y$; il correspond donc à une classe $\alpha \in \mathrm{H}^{1}(Y$, Aut $G)$.

On suppose en outre qu'il existe un $k$-schéma en groupes lisse $\Gamma$ et un $k$-morphisme $\varphi: \Gamma \rightarrow$ Aut $G$ tels que $\alpha$ soit l'image d'une classe $\gamma \in \mathrm{H}^{1}(Y, \Gamma)$ par $\mathrm{H}^{1}(Y, \varphi): \mathrm{H}^{1}(Y, \Gamma) \rightarrow \mathrm{H}^{1}(Y$, Aut $G)$.

Alors, pour tout $y \in Y(k)$, il existe un voisinage étale pointé $\left(Y^{\prime}, y^{\prime}\right)$ de y tel que le $Y^{\prime}$-groupe $\mathfrak{G} \times_{Y} Y^{\prime}$ soit constant (et donc isomorphe à $Y^{\prime} \times_{k} \mathfrak{G}_{y}$ où $\mathfrak{G}_{y}$ est la fibre de $\mathfrak{G}$ en $y$ ).

Démonstration. La classe $\gamma$ correspond à un $\Gamma$-torseur $T \rightarrow Y$. Soit $T_{1} \rightarrow Y$ le $\Gamma$-torseur $Y \times_{k} T_{y}$ où $T_{y}$ est la fibre de $T$ en $y$. Alors $U:=\underline{\operatorname{Isom}}_{\Gamma}\left(T, T_{1}\right)$ est un torseur sous $\underline{A u t}_{\Gamma}(T)$ qui est une forme de $\Gamma$ et donc un $Y$-espace en groupes lisse. Donc $U$ est un $Y$-espace algébrique lisse sur $Y$. Comme il a un point rationnel au-dessus de $y$, il admet donc une section sur un voisinage étale pointé $\left(Y^{\prime}, y^{\prime}\right)$ de $y$. Donc $T$ devient constant sur $Y^{\prime}$, c'est-à-dire que $\gamma_{Y^{\prime}}$ provient d'une classe dans $\mathrm{H}^{1}(k, \Gamma)$, d'où il suit que $\alpha_{Y^{\prime}}$ provient d'une classe de $\mathrm{H}^{1}(k, \underline{\text { Aut }} G)$, d'où la conclusion.

7.4.4 Corollaire. Sous les hypothèses du lemme 7.4.3, on suppose en outre que $Y$ est localement séparé, que $k$ est un corps topologiquement hensélien, et que :

- ou bien $\mathfrak{G}$ est lisse sur $Y$ (ou encore que $G$ est un $k$-groupe lisse, ce qui revient au même si $Y \neq \emptyset)$;

- ou bien $k$ est un corps valué admissible.

Soit $f: X \rightarrow Y$ un $\mathfrak{G}$-torseur. Alors $\operatorname{Im}\left(f_{\text {top }}\right) \subset Y_{\text {top }}$ est localement fermée, et l'application induite $X_{\text {top }} \rightarrow \operatorname{Im}\left(f_{\text {top }}\right)$ est une $\mathfrak{G}_{\text {top }}$-fibration principale.

De plus $\operatorname{Im}\left(f_{\text {top }}\right)$ est ouverte et fermée si $\mathfrak{G}$ est lisse, et est fermée si $G$ vérifie $\left.{ }^{*}\right)$.

Démonstration. La question étant locale sur $Y_{\text {top }}$, le lemme 7.4 .3 ramène la situation au cas

d'un $Y$-groupe constant (l'hypothèse sur $Y$ assure qu'avec les notations du lemme, $Y_{\text {top }}^{\prime} \rightarrow Y_{\text {top }}$ est un homéomorphisme local). On applique alors le théorème 1.2 dans le cas admissible, et la proposition 3.4.1 dans le cas lisse.

7.4.5 Remarques. La condition du lemme 7.4.3 sur l'existence de $\varphi: \Gamma \rightarrow \underline{\text { Aut }} G$ est très restrictive. Elle est trivialement vérifiée si Aut $G$ est représentable et lisse, notamment lorsque $\mathfrak{G}$ est réductif [SGA3, XXIV, théorème 1.3]; noter que dans ce cas Aut $G$ n'est pas toujours de type fini.

Un autre cas utile est celui des « $Y$-formes fortement intérieures 》 (voir par exemple [CF14, 2.2.4.9] ; certains auteurs les appellent « formes intérieures pures ») d'un $k$-groupe algébrique $G$ : partant d'un $G$-torseur (à droite) $X_{1} \rightarrow Y$, on considère le $Y$-groupe $\mathfrak{G}:=\underline{\operatorname{Aut}}_{G}\left(X_{1}\right)$. Sa classe dans $\mathrm{H}^{1}(Y, \underline{\text { Aut }} G)$ est l'image de la classe de $X_{1}$ par

$$
\mathrm{H}^{1} \text { (int) }: \mathrm{H}^{1}(Y, G) \rightarrow \mathrm{H}^{1}(Y, \underline{\text { Aut }} G),
$$

où int $: G \rightarrow \underline{\text { Aut }} G$ est le morphisme de conjugaison. Ce dernier passe au quotient par le centre $Z(G)$ de $G$, de sorte que le lemme 7.4.3 et le corollaire 7.4.4 s'appliquent à $\mathfrak{G}$ chaque fois que $G / Z(G)$ est lisse. Comme tout $\mathfrak{G}$-torseur à droite est de la forme $\underline{\operatorname{Isom}}_{G}\left(X_{1}, X_{2}\right)$ où $X_{2}$ est un autre $G$-torseur sur $Y$, on obtient la proposition 7.4.6 qui suit.

7.4.6 Proposition. Soient $(K, v)$ un corps valué admissible et $Y$ un $K$-espace localement séparé. Soit $G$ un $K$-groupe algébrique. On suppose que $G / Z(G)$ est lisse.

Soient $X_{1} \rightarrow Y$ et $X_{2} \rightarrow Y$ des $G$-torseurs. On note $\mathfrak{G}_{i}:=\underline{\text { Aut }}_{G}\left(X_{i}\right) \rightarrow Y$ le tordu intérieur de $G$ par le torseur $X_{i}(i=1,2)$. On considère le $\left(\mathfrak{G}_{1}, \mathfrak{G}_{2}\right)$-bitorseur $f: T \rightarrow Y$ défini par

$$
T:=\underline{\operatorname{Isom}}_{G}\left(X_{1}, X_{2}\right) .
$$




\section{Ofer Gabber, Philippe Gille et Laurent Moret-Bailly}

On désigne par I l'image de $f_{\text {top }}: T_{\text {top }} \rightarrow Y_{\text {top }}$.

(1) I est localement fermé dans $Y_{\mathrm{top}}$, et est fermé si $G$ vérifie $(*)$.

(2) Pour tout $y \in I$, il existe un voisinage ouvert $U_{y}$ de $y$ dans $I$ de sorte que l'application $f_{\text {top }}^{-1}\left(U_{y}\right) \rightarrow U_{y}$ soit munie d'une structure de $\left(G_{y}\right)_{\text {top }}$-fibré principal, où $G_{y}=\mathfrak{G}_{2} \times_{Y} y$.

7.4.6.1 Remarque. Les deux assertions de 7.4.6 sont en défaut si l'on omet l'hypothèse que $G / Z(G)$ est lisse. On peut en effet construire un exemple (dérivé de 7.1), avec $G=\alpha_{p} \rtimes_{K} \mathbb{G}_{m}$, où $I$ n'est pas localement fermé, et où $f_{\text {top }}$ n'est pas stricte.

\section{REMERCIEMENTS}

Nous tenons à remercier Brian Conrad et Bertrand Lemaire pour leurs suggestions bienvenues, et le rapporteur pour sa lecture attentive du manuscrit et ses nombreuses remarques.

\section{REFERENCES}

Art69 M. Artin, Algebraization of formal moduli: I, in Global Analysis (Papers in honor of K. Kodaira), University of Tokyo Press, Tokyo, 1969.

Art74 M. Artin, Versal Deformations and Algebraic Stacks, Invent. Math. 27 (1974), 165-189.

BaT13 D. P. Bac and N. Q. Thang, On the topology of relative and geometric orbits for actions of algebraic groups over complete fields, J. of Algebra 390 (2013), 181-198. http://dx.doi.org/ $10.1016 / \mathrm{j}$.jalgebra.2013.04.040

Ber09 J. Bertin, The punctual Hilbert scheme, an introduction, available at https://cel. archives-ouvertes.fr/cel-00437713

BLR90 S. Bosch, W. Lütkebohmert and M. Raynaud, Néron models, Ergebnisse der Mathematik und ihrer Grenzgebiete 21, Springer-Verlag, Berlin, 1990.

BoT65 A. Borel and J. Tits, Groupes réductifs, Publ. Math. Inst. Hautes Études Sci. 27 (1965), 55-150.

Bou71 N. Bourbaki, Topologie générale, chapitres 1 à 4, Hermann, Paris, 1971.

Bou75 N. Bourbaki, Algèbre commutative, chapitres 5 à \%, Springer-Verlag, Berlin, 1975

BZ76 I. N. Bernstein and A. V. Zelevinskiř, Representations of the group $G L(n, F)$, where $F$ is a local non-Archimedean field, Uspekhi Mat. Nauk 31 (1976), 5-70.

CF14 B. Calmès and J. Fasel, Groupes classiques, arXiv:1401.1992 (2014).

CGP10 B. Conrad, O. Gabber and G. Prasad, Pseudo-reductive groups, Cambridge University Press, Cambridge, 2010. http://dx.doi.org/10.1017/CB09780511661143

Cho57 W. L. Chow, On the projective embedding of homogeneous varieties, A symposium in honor of S. Lefschetz, p. 122-128, Princeton University Press, Princeton, NJ, 1957.

CLO09 B. Conrad, M. Lieblich and M. Olsson, Nagata compactification for algebraic spaces, arXiv:0910.5008v2.

Con07 B. Conrad, Deligne's notes on Nagata compactifications, J. Ramanujan Math. Soc. 22 (2007), no $3,205-257$.

Con12 B. Conrad, Weil and Grothendieck Approaches to Adelic Points, Enseign. Math. (2) 58 (2012), 61-97. http://dx.doi.org/10.4171/LEM/58-1-3

DG70 M. Demazure and P. Gabriel, Groupes algébriques, North-Holland, Amsterdam; Masson \& Cie., Paris, 1970.

EGAI A. Grothendieck and J. Dieudonné, Éléments de géométrie algébrique I, Grundlehren der Mathematischen Wissenschaften 166, Springer (1971). 


\section{FIBRÉS PRINCIPAUX SUR LES CORPS VALUÉS HENSÉLIENS}

EGAII A. Grothendieck and J. Dieudonné, Éléments de géométrie algébrique II, Publ. Math. Inst. Hautes Études Sci. 8 (1961).

EGAIV A. Grothendieck and J. Dieudonné, Éléments de géométrie algébrique IV.2, IV.3, and IV.4, Publ. Math. Inst. Hautes Études Sci. 24, 28 (1965) and 32 (1967).

Gab12 O. Gabber, On pseudo-reductive groups and compactification theorems, Oberwolfach Rep. (2012), 2371-2374.

Gir71 J. Giraud, Cohomologie non abélienne, Grundlehren der Mathematischen Wissenschaften 179, Springer-Verlag, Berlin-New York, 1971.

GR71 L. Gruson and M. Raynaud, Critères de platitude et de projectivité, Invent. Math. 13 (1971), $1-89$.

Gre66 M. J. Greenberg, Rational points in Henselian discrete valuation rings, Publ. Math. Inst. Hautes Études Sci. 31 (1966), 59-64.

Gro60 A. Grothendieck, Techniques de construction et théorèmes d'existence en géométrie algébrique IV: les schémas de Hilbert, Séminaire Bourbaki 6 (1960-1961), Exp. No. 221, 249-276, Soc. Math. France, Paris, 1995.

Gro68 A. Grothendieck, Le groupe de Brauer III, Dix exposés sur la cohomologie des schémas, Advanced Studies in Pure Mathematics, North-Holland, Amsterdam; Masson, Paris, 1968.

GW10 U. Görtz and T. Wedhorn, Algebraic geometry I. Schemes with examples and exercises, Advanced Lectures in Mathematics, Vieweg and Teubner, Wiesbaden, 2010. http://dx.doi.org/ 10.1007/978-3-8348-9722-0

Knu71 D. Knutson, Algebraic Spaces, Lecture Notes in Math. 203, Springer-Verlag, Berlin-New York, 1971.

Kol92 J. Kollár, Cone theorems and bug-eyed covers, J. Algebraic Geom. 1 (1992), 293-323.

KS83 K. Kato and S. Saito, Unramified class field theory of arithmetical surfaces, Ann. of Math. 118 (1983), 241-275.

Kuh F.-V. Kuhlmann, Valuation Theory, available at http://math.usask.ca/ fvk/Fvkbook.htm.

LMB00 G. Laumon and L. Moret-Bailly, Champs algébriques, Ergebnisse der Mathematik und ihrer Grenzgebiete. 3. Folge A Series of Modern Surveys in Mathematics, Vol. 39 , Springer-Verlag, Berlin, 2000.

MB12a L. Moret-Bailly, Un théorème de l'application ouverte sur les corps valués algébriquement clos, Math. Scand. 111 (2012), 161-168.

MB12b L. Moret-Bailly, An extension of Greenberg's theorem to general valuation rings, Manuscripta Math. 139 (2012), no 1, 153-166. http://dx.doi.org/10.1007/s00229-011-0510-5

Mil80 J. S. Milne, Étale cohomology, Princeton Mathematical Series 33, Princeton University Press, Princeton, NJ, 1980.

Moc99 S. Mochizuki, Foundations of p-adic Teichmüller theory, Studies in Advanced Mathematics 11, American Mathematical Society, Providence, RI; International Press, Cambridge, MA, 1999.

Nag62 M. Nagata, Local Rings, Interscience Tracts in Pure and Applied Mathematics No. 13, Interscience Publishers, New York-London, 1962.

Nit05 N. Nitsure, Construction of Hilbert and Quot schemes, dans Fundamental Algebraic Geometry: Grothendieck's FGA Explained, Math. surveys and Monographs 123, Amer. Math. Soc., Providence, RI, 2005.

Ols06 M. C. Olsson, Hom-stacks and restriction of scalars, Duke Math. J. 134 (2006), 139-164. http: //dx.doi.org/10.1215/S0012-7094-06-13414-2

PZ78 A. Prestel and M. Ziegler, Model theoretic methods in the theory of topological fields, J. Reine Angew. Math. 299(300) (1978), 318-341.

Ray70 M. Raynaud, Faisceaux amples sur les schémas en groupes et les espaces homogènes, Lecture Notes in Math. 119, Springer-Verlag, Berlin-New York, 1970. 
Sch02 S. Schröer, Hilbert's Theorem 90 and Algebraic Spaces, J. Pure Appl. Algebra 173 (2002), 339-345. http://dx.doi.org/10.1016/S0022-4049(02)00012-9

Ser94 J.-P. Serre, Cohomologie galoisienne, Fifth edition, Lecture Notes in Mathematics 5, SpringerVerlag, Berlin, 1994.

SGA1 Séminaire de Géométrie algébrique du Bois-Marie, Revêtements étales et groupe fondamental, dirigé par A. Grothendieck, Documents Mathématiques vol. 3, Société mathématique de France, Paris, 2003.

SGA3 Séminaire de Géométrie algébrique du Bois-Marie, 1962-1964, Schémas en groupes, dirigé par M. Demazure et A. Grothendieck, Documents Mathématiques vol. 7 and 8, Société mathématique de France, Paris, 2011.

Tho83 R. Thomason, Algebraic K-theory of group scheme actions, Algebraic Topology and algebraic K-theory (Princeton, NJ, 1983), 539-563, Ann. of Math. Studies 113, Princeton University Press, Princeton, NJ, 1987.

War89 S. Warner, Topological fields, North-Holland Mathematics Studies 157, North-Holland Publishing Co., Amsterdam, 1989.

Wei62 A. Weil, Foundations of Algebraic Geometry, American Mathematical Society, Providence, RI, 1962 .

Ofer Gabber gabber@ihes.fr

C.N.R.S. and I.H.É.S., Le Bois-Marie, 35 route de Chartres, 91440 Bures sur Yvette, France

Philippe Gille gille@math.univ-lyon1.fr

UMR 5208 du CNRS, Institut Camille Jordan, Université Claude Bernard Lyon 1, 43 boulevard du 11 novembre 1918, 69622 Villeurbanne Cedex, France

Laurent Moret-Bailly laurent.moret-bailly@univ-rennes1.fr

IRMAR, Université de Rennes 1, Campus de Beaulieu, 35042 Rennes Cedex, France 